Notre Dame Journal of Formal Logic

Volume 30, Number 1, Winter 1989

\title{
Multiset Theory
}

\author{
WAYNE D. BLIZARD*
}

A multiset is a collection of objects (called elements) in which elements may occur more than once. The number of times an element occurs in a multiset is called its multiplicity. The cardinality of a multiset is the sum of the multiplicities of its elements. Multisets are of interest in certain areas of mathematics, computer science, physics, and philosophy. Section 1 introduces multisets and surveys the relevant literature. Section 2 develops a first-order two-sorted theory MST for multisets that "contains" classical set theory. The intended interpretation of the atomic formula $x \in^{n} y$ is " $x$ is an element of $y$ with multiplicity $n$ ". In MST, one can extend the classical notion of a function. Section 3 constructs a model of MST in ZFC by interpreting $x \in^{n} y$ as $y(x)=n$ (multisets are modeled by positive integer-valued functions).

Introduction In [16] Kamke spells out the assumptions underlying classical set theory and thereby classical mathematics as a whole:

By a set we are to understand, according to G. Cantor, "a collection into a whole, of definite, well-distinguished objects (called the 'elements') of our perception or of our thought . . .". For a set, the order of succession of its elements shall not matter. . . . Furthermore, the same element shall not be allowed to appear more than once. The number complex $1,2,1,2,3$, consequently, becomes a set only after deleting the repeated elements.

\footnotetext{
*This paper is based on part of the author's doctoral thesis prepared under the supervision of Dr. Robin Gandy at the University of Oxford. The research was supported by a Natural Sciences and Engineering Research Council of Canada Scholarship and a United Kingdom Overseas Research Student Award.

I would like to thank my supervisor, Dr. Robin Gandy, and my examiners, Dr. Dan Isaacson and Professor T. J. Smiley. I thank everyone with whom I have discussed multisets and their possible applications, especially T. Hailperin, G. Miller, A. F. Parker-Rhodes, and R. Rado; also M. Hallett, J. Hickman, J. Hoskins, P. Koepke, S. Lee, Z. Manna, B. Müller, M. Osterheld, F. Sheridan, W. van Stigt, and R. Walker. I also thank the referee for valuable comments, especially his suggestion to modify the $H$ operation which simplified the definition of cardinality.
} 
A multiset is a collection of elements in which elements are allowed to repeat; it may contain a finite number of indistinguishable copies of a particular element. A set is a multiset in which distinct elements occur only once. The number of times an element occurs in a multiset (its multiplicity) is relevant, and each occurrence contributes to the cardinality of the multiset. The number of distinct elements in a multiset need not be finite.

The word "multiset" (often shortened to mset) which abbreviates the term "multiple-membership set", is now the commonly accepted name for this concept, replacing "bag", "bunch", "weighted set", "occurrence set”, "heap", "sample", and "fireset"-finitely repeated element set.

Our survey of the literature indicates that multisets arise quite naturally in certain areas of mathematics, computer science, physics, and philosophy. In classical mathematics, however, one cannot deal directly with multisets. If one wishes to use collections of sets with repetitions, one is forced to consider sequences of sets, or more generally, families of sets. One could also define a multiset which contains the element $x$ with multiplicity $n$ to be a function which contains the ordered pair $\langle x, n\rangle$. However, as Knuth points out ([17], p. 636) “. . . this formal equivalence is of little or no practical value for creative mathematical reasoning." It is often preferable in many applications to deal directly with multiple-membership sets. Knuth concludes ([17], p. 636) that "Although multisets appear frequently in mathematics, they often must be treated rather clumsily because there is currently no standard way to treat sets with repeated elements. Several mathematicians have voiced their belief that the lack of adequate terminology and notation for this common concept has been a definite handicap to the development of mathematics." In [23] Meyer and McRobbie argue that the lack of an adequate theory of multisets has also impeded the development of logic and philosophy. In Section 2 we develop a theory MST for multisets that "extends" classical set theory-MST contains a copy of ZFC.

1 A survey of the literature Multisets can be traced back to the very origins of the concept of number. In ancient times, the number $n$ was often represented by a collection of $n$ strokes, tally marks, or units. Thus, for example, the number seven came to be identified with a collection of objects like /////// ([13], p. 132). One can develop ([3], pp. 26-27) an algebra of simple multisets of 1's that corresponds exactly to the additive arithmetic of the natural numbers. By identifying a positive integer with the unique multiset of its prime factors, one can also develop an algebra of multisets that corresponds to the multiplicative arithmetic of the positive integers ([17], p. 464; [3], pp. 40-43).

With respect to the notion of multiplicity, there is a philosophical view that states that the plurality in things arises from their diversity. Frege remarks that "Number is but another name for diversity. Exact identity is unity, and with difference arises plurality" ([11], s. 35). Leibniz states ". . . it is not true that two substances resemble each other entirely and are different in number alone ...." ([20], p. 19). The naive concept of multiset takes a different view. Occurrences of an element $x$ in a multiset cannot be distinguished. The cardinality of a multiset (the sum of the multiplicities of its elements) assumes "plurality without diversity". Indeed, classical mathematics accepts the principle of plurality with- 
out diversity. In algebra, for example, repeated roots of polynomials, although identical in all respects, are treated as a multiplicity. The polynomial $x^{2}-$ $2 x+1=(x-1)^{2}$ is said to have two factors and two roots. Repeated observations in a statistical sample are counted as a multiplicity, each repetition contributing to a calculated probability.

Many references to multisets can be found in the literature of classical mathematics. In [6] Dedekind observes that each element in the range of a function can be thought of as having a multiplicity equal to the number of elements in the domain that are mapped to it. He concludes, "In this way we reach the notion, very useful in many cases, of systems in which every element is endowed with a certain frequency-number which indicates how often it is to be reckoned as an element of the system" ([6], p. 114).

Weierstrass defined real numbers as certain multisets of rational numbers ([4], pp. 18-20; [13], p. 134). A quick way to see this (a simplification of Weierstrass's approach) is to identify, for example, the real number $\pi=3.141 \ldots$ with the multiset that contains the element 1 with multiplicity 3 , the element $\frac{1}{10}$ with multiplicity 1 , the element $\frac{1}{100}$ with multiplicity 4 , etc. The rational sum of the elements of the multiset (with repetitions counted) equals $\pi$.

It was Cantor's intention that 'aggregates' consist only of distinct elements. Yet Cantor himself defined cardinality as a collection of repeated 'ones' and he referred to such collections as 'definite aggregates' ([4], p. 86). This would seem to contradict Cantor's restriction on membership - "definite, distinct objects"([4], p. 85) in the same 1895 paper.

Weyl in Appendix B of [27] makes use of multisets: “. . . an aggregate of white, red, and green balls may contain several white balls. Generally speaking, in a given aggregate there may occur several individuals, or elements, of the same kind (e.g., several white balls) or, as we shall also say, the same entity (e.g., the entity white ball) may occur in several copies" ([27], p. 238). Weyl applies his notion of multiset (a set with an equivalence relation) to a variety of problems in physics, chemistry, and genetics. The equivalence relation approach to multisets has also been investigated in Monro's [24] which is motivated primarily by concepts from category theory.

Developments in physics led Parker-Rhodes to define an elaborate mathematical system (a theory of 'sorts') to deal with collections of indistinguishable objects [25]. Elements of multisets conform to the Parker-Rhodes principle of indistinguishables - copies of elements behave as identicals in isolation, but contribute 'severally' to cardinality when they belong to the same multiset. The system of Parker-Rhodes differs radically from classical mathematics, whereas the theory MST 'extends' and 'contains' classical set theory.

Levy uses multisets ([21], p. 100) as a conceptual aid while discussing cardinal arithmetic. Although their conceptual advantages are admitted, Levy does not deal directly with multisets. Hailperin's [12] uses 'heaps' to interpret the logical system used in G. Boole's Laws of Thought. Knuth finds multisets useful for defining numerical algorithms ([17], p. 441) and for a variety of applications in computer search-and-sort procedures [18]. Dershowitz and Manna [7] use multisets to prove that certain types of computer programs terminate. In [15] Hickman defines multisets as cardinal-valued functions in ZFC and develops their formal algebra while emphasizing the differences between multisets and 
classical sets. Meyer and McRobbie [23] give ". . . the first sustained investigation of multisets in a logical and philosophical context ..." noting that “. . . computer scientists and mathematicians have been relatively slow in adding multisets to their technical vocabulary" ([23], notes 3, 4, p. 107). They demonstrate that multisets have the appropriate degree of abstraction (between sets and sequences) needed to study relevant implication.

In [28] Whitney investigates the algebraic properties of 'generalized sets' ('sets' whose characteristic functions have ranges more general than $\{0,1\}$ ), “. . . where each element is counted any number of times" ([28], p. 412). Whitney claims that 'generalized sets' are useful in various mathematical theories citing "chains in analysis situs" as one example ([28], pp. 405, 412). Multisets are used by Rado in [26] to study families of sets. Rado notes that "The notion of a set takes no account of multiple occurrence of any one of its members, and yet it is just this kind of information which is frequently of importance. We need only think of the set of roots of a polynomial $f(x)$ or the spectrum of a linear operator" ([26], p. 135).

In Chapter 11 of [22] Manna and Waldinger develop an elementary theory of bags using a primitive binary insertion symbol $\odot$. If an atom $u$ has multiplicity $n \geq 0$ in bag $x$, then $u$ has multiplicity $n+1$ in bag $u \odot x$. Their theory BAG admits only finite collections of atoms (no hierarchy of bags) and is developed to the point of a simple algebra of bags.

Lake in [19] proposes an axiomatization for multisets based on von Neumann's 1925 axiom system for set theory which takes the notion of function as primitive. We have rejected this approach for several reasons. It is well known that von Neumann's 1925 axiomatization of set theory automatically gives the axiom of choice. However, one may wish to work without the axiom of choice. Fraenkel, Bar-Hillel, and Levy characterize von Neumann's approach (in spite of later simplifications) as "rather clumsy" and conclude that ". . . it is after all simpler to take the notions of set and class, or only that of class alone, as the basic notions of set theory" ([10], p. 135). Indeed, Lake admits that “. . . it might be thought desireable to have an axiomatization which does not go via functions. Such an axiomatization . . . could be conveniently written out using $x \in_{z} y$ (formally a three place predicate) to stand for ' $x$ belongs to $y$ precisely $z$ times' '([19], p. 325). We use a primitive ternary membership relation $e$ to define the theory MST.

2 A theory of multisets The naive concept of multiset that we now formalize has the following properties: (i) a multiset is a collection of elements in which certain elements may occur more than once; (ii) occurrences of a particular element in a multiset are indistinguishable; (iii) each occurrence of an element in a multiset contributes to the cardinality of the multiset; (iv) the number of occurrences of a particular element in a multiset is a (finite) positive integer; (v) the number of distinguishable (distinct) elements in a multiset need not be finite; and (vi) a multiset is completely determined if we know which elements belong to it and the number of times each element belongs to it.

The theory MST is formulated in the first-order predicate calculus with equality using conventional logical symbols: $\sim$ (not), $\wedge$ (and), $\vee($ or $), \rightarrow$ 
(if . . . then ...), $\leftrightarrow$ (iff), $\forall$ (for all), $\exists$ (there exists), $=_{M}$ (equality for multisets), $=_{N}$ (equality for numbers), and enclosures (, ), ], [.

The first-order language $L$ of MST employs two sorts of variable symbols: mset variable symbols $x, y, z, \ldots$ used to denote msets and elements of msets, and numerical variable symbols $\dot{k}, \dot{l}, \dot{m}, \dot{n}, \ldots$ used to denote multiplicities of elements in msets. The two sorts of variables of $L$ are intended to range over disjoint universes: $\mathrm{M}$ (for multisets) and $\mathrm{N}$ (for numbers). Each sort has its own equality sign: $={ }_{M}$ (of sorting $\mathrm{M} \times \mathrm{M}$ ) and $=_{N}$ (of sorting $\mathrm{N} \times \mathrm{N}$ ). The twosorted language $L$ is used for convenience only. There is nothing essential that can be done with $L$ that cannot be done with a first-order one-sorted language ([8], pp. 277-281; [1], p. 42).

The nonlogical symbols of $L$ are $\{e, \hat{,}, S,+, \cdot, 0\}$ where $e$ is a ternary membership relation symbol of sorting $\mathrm{M} \times \mathrm{M} \times \mathrm{N}$, is a unary function symbol of sorting $\mathrm{N} \rightarrow \mathrm{M}, \mathrm{S}$ is a unary function symbol of sorting $\mathrm{N} \rightarrow \mathrm{N},+$ and $\cdot$ are binary function symbols of sorting $\mathrm{N} \times \mathrm{N} \rightarrow \mathrm{N}$ and 0 is a numeric constant symbol.

An expression of $L$ is any finite sequence of symbols of $L$. The collection of numeric terms of $L$ is the smallest collection of expressions of $L$ that contains the numeric variable symbols, the constant symbol 0 , and is closed under the function symbols $\mathrm{S}, \cdot$, and + . We use metamathematical symbols $s$ and $t$ to denote numeric terms of $L$. If $s$ and $t$ are numeric terms of $L$, then $S t, s \cdot t$, and $s+t$ are also numeric terms of $L$. The mset terms of $L$ are the mset variable symbols of $L$ together with all expressions of $L$ of the form $\hat{t}$ where $t$ is any numeric term of $L$. It will turn out that $\hat{t}$ is just the usual von Neumann set associated with the number $t$. We use metamathematical symbols $u, v$, and $w$ to denote mset terms of $L$. The terms of $L$ are the numeric terms of $L$ together with the mset terms of $L$. Terms of $L$ name objects - they do not make assertions about objects.

The atomic formulas of $L$ are all expressions of $L$ of the forms $s={ }_{\mathrm{N}} t$, $u={ }_{\mathrm{M}} v$, and $e(u, v, t)$. The fact that each sort has its own equality sign automatically ensures that a numeric term cannot be equated to a multiset term. Expressions like $e(x, \dot{m}, \dot{n}), e(\dot{m}, y, \dot{n}), e(\dot{k}, \dot{m}, \dot{n}), e(\dot{n}, \dot{m}, x)$, and $e(x, y, \hat{\dot{n}})$ are not well-formed in $L$ since they cannot arise from the substitution of equals in atomic formulas. For simplicity we will use the single equality symbol $=$. It will be obvious from the context whether $=_{\mathrm{N}}$ or $=_{\mathrm{M}}$ is intended.

The intended interpretation of the atomic formula $e(u, v, t)$ is " $u$ is an element of the mset $v$ with multiplicity exactly $t$." We introduce the dressed ternary epsilon relation by the definition: $u \in \in^{t} v$ stands for $e(u, v, t)$ for any mset terms $u$ and $v$ and any numeric term $t$. From this point onward, we drop the symbol $e$ and use the ternary epsilon symbol instead.

The well-formed-formulas, $w f f s$, of $L$ are defined as follows: all atomic formulas of $L$ are wffs, and if $\varphi$ and $\psi$ are wffs, then so are $\sim \varphi, \varphi \wedge \psi, \varphi \vee \psi$, $\varphi \rightarrow \psi, \varphi \leftrightarrow \psi$; and for all variable symbols $x$ and $\dot{n}$, so are $\exists x \varphi, \forall x \varphi, \exists \dot{n} \varphi$, and $\forall \dot{n} \varphi$.

We introduce the following shorthand notation for numeric quantifiers: for any wff $\varphi$ of $L, \forall n \varphi$ stands for $\forall \dot{n}(\dot{n} \neq 0 \rightarrow \varphi)$ and $\exists n \varphi$ stands for $\exists \dot{n}(\dot{n} \neq$ $0 \wedge \varphi$ ). We do this to avoid writing $\dot{n} \neq 0$ in almost every formula.

We introduce the undressed binary epsilon relation by the definition: 
for any mset terms $u$ and $v, u \in v$ stands for $\exists n u \in \in^{n} v$ and $u \notin v$ stands for $\sim \exists n u \in^{n} v$ or $\forall n \sim u \in^{n} v$. The intended interpretation, therefore, of $u \in v$ is " $u$ is an element of the mset $v$ with some nonzero multiplicity". Thus, $u \in v$ is a wff of $L$ and not an atomic formula of $L$.

The variable symbol $x$ (or $n$ ) is said to be bound in a wff $\varphi$ if it lies within the scope of quantifiers $\forall x$ or $\exists x$ (or $\forall n$ or $\exists n$ ) in $\varphi$. A variable symbol occurs free in a wff $\varphi$ if it is not bound in $\varphi$. We assume that a variable symbol does not occur both bound and free in a wff (bound variable symbols can always be replaced by variable symbols that do not occur in the wff). A wff $\varphi$ is an $L$ sentence if there are no variable symbols (mset or numeric) occurring free in $\varphi$.

As in [21], p. 5, we adopt the convention that $\varphi(\ldots)$ means that the interesting cases of what is to be said are those where the variable symbols displayed in the list . . a are free in $\varphi$. Therefore, $\varphi(x)$ (or $\varphi(n)$ ) does not mean that $x$ (or $n)$ is a free variable of $\varphi$, nor does it mean that $\varphi(x)$ (or $\varphi(n))$ has no free variables other than $x$ (or $n)$. When $\varphi(u)$ (or $\varphi(t)$ ) is used after first using the notation $\varphi(x)$ (or $\varphi(n)$ ), we mean the wff obtained from $\varphi(x)$ (or $\varphi(n))$ by the "proper" substitution of the mset term $u$ (or the numeric term $t$ ) for the free occurrences of $x$ (or $n$ ) if any. By "proper" substitution we mean that we assume that collisions between variable symbols are avoided. So, for example, if $n$ is bound in $\varphi(x)$, then we assume that the bound occurrences of $n$ are replaced by some other suitable symbol $n^{\prime}$ before $\hat{n}$ is substituted for free occurrences of $x$ to obtain the wff $\varphi(\hat{n})$.

By an $L$-theory we mean the collection of all $L$-sentences that are the logical consequences generated from a collection of axioms. The axioms of the $L$-theory MST are the axioms numbered N1 through N8 and I through XII described below.

If $T$ is a theory, we write $T \vdash \varphi$ to mean the sentence $\varphi$ is in $T$ ( $\varphi$ is a theorem of $T$, or $\varphi$ is provable from the axioms of $T$ ). As is normal practice, we give informal proofs of theorems. We write $\vdash \varphi$ if $\varphi$ is a logical truth. If class terms of the form $\{u \mid \varphi(u)\}$ or $\{t \mid \psi(t)\}$ are used, the expressions in which they occur are always reducible to wffs in $L$ (see, for example, 3.1 of [21], p. 9).

The formal theory of vector spaces is a two-sorted first-order theory that assumes the theory of fields for its scalar variables. Although not always made explicit, the axioms of the theory of vector spaces include the axioms of the theory of fields. In exactly the same way, MST is a two-sorted first-order theory that assumes the theory of arithmetic for its numeric variables. The axioms of MST, therefore, include the following axioms of Peano Arithmetic (adapted from [5], p. 42):

$$
\begin{array}{ll}
\text { N1 } & \forall \dot{n}(\sim \mathrm{S} \dot{n}=0) \\
\text { N2 } & \forall \dot{n} \forall \dot{m}(\mathrm{~S} \dot{n}=\mathrm{S} \dot{m} \rightarrow \dot{n}=\dot{m}) \\
\text { N3 } & \forall \dot{n}(\dot{n}+0=\dot{n}) \\
\text { N4 } & \forall \dot{n} \forall \dot{m}((\dot{n}+\mathrm{S} \dot{m})=\mathrm{S}(\dot{n}+\dot{m})) \\
\text { N5 } & \forall \dot{n}(\dot{n} \cdot 0=0) \\
\text { N6 } & \forall \dot{n} \forall \dot{m}(\dot{n} \cdot \mathrm{S} \dot{m}=\dot{n} \cdot \dot{m}+\dot{n}) .
\end{array}
$$

The axiom schema of induction states: for every wff $\varphi(\dot{n})$ of $L$, the universal closure of 
N7 $\varphi \quad(\varphi(0) \wedge \forall \dot{n}(\varphi(\dot{n}) \rightarrow \varphi(\mathrm{S} \dot{n}))) \rightarrow \forall \dot{n} \varphi(\dot{n})$

is an axiom of MST.

The theory PA (Peano Arithmetic) has the first-order language $\{\mathrm{S},+, \cdot, 0\}$ with only the numeric variable symbols. The axioms of PA are Axioms N1 through N6 and all Axioms $\mathrm{N}_{\varphi}$ where $\varphi$ is a wff of the language $\{\mathrm{S},+, \cdot, 0\}$. It should be noted that Axioms $\mathrm{N}_{\varphi}$ as stated above are stronger than the induction schema for PA since the wff $\varphi$ may contain multiset terms.

For numeric terms $s$ and $t$ of $L$, we define

$s<t$ stands for $\exists n(t=s+n)$

$s \leq t$ stands for $\exists \dot{n}(t=s+\dot{n})$.

In PA, it is well known that trichotomy and the least number principle hold. In MST, therefore,

$$
\forall \dot{n} \forall \dot{m}(\dot{n}<\dot{m} \vee \dot{n}=\dot{m} \vee \dot{m}<\dot{n}) \text { holds }
$$

and, for every wff $\varphi(\dot{n})$ of $L$,

$$
\exists \dot{n} \varphi(\dot{n}) \rightarrow \exists \dot{n}(\varphi(\dot{n}) \wedge \forall \dot{m}(\dot{m}<\dot{n} \rightarrow \sim \varphi(\dot{m}))) \text { holds. }
$$

We give names to some special numeric terms of $L$ by defining: 1 stands for S0, 2 stands for $\mathrm{SS} 0$, etc. The class term $\mathrm{N}=\{\dot{n} \mid \dot{n}=\dot{n}\}$ is called the numeric universe of MST. If we write $\dot{m} \in\{\dot{n} \mid \psi(\dot{n})\}$ to mean $\psi(\dot{m})$, then $\forall \dot{n} \dot{n} \in \mathrm{N}$.

The theory PA may have nonstandard models. In a nonstandard model of MST (a model of MST in which the numeric universe is nonstandard) multiplicities of elements may be infinite in the model. The set $\hat{n}$ is the usual von Neumann numeral associated with the number $n$. If $n$ is nonstandard in the model, then $\hat{n}$ will be a non-well-founded set in the model. The existence of nonstandard models of MST need not concern us in the development of the theory itself.

The exact multiplicity axiom of MST is

I $\forall x \forall y \forall n \forall m\left(\left(x \in^{n} y \wedge x \in^{m} y\right) \rightarrow n=m\right)$.

In other words, the multiplicity with which an element belongs to a multiset is unique.

The axiom of extensionality of MST is

II $\forall x \forall y\left(\forall z \forall n\left(z \in^{n} x \leftrightarrow z \in^{n} y\right) \rightarrow x=y\right)$.

In other words, if two msets have exactly the same elements occurring with exactly the same multiplicities, then they are equal. The converse of Axiom II follows by the substitutivity of = in our logic.

The empty set axiom of MST is

III $\exists y \forall x \forall n \sim x \in^{n} y$.

It asserts the existence of at least one mset that does not contain any elements. In fact, the mset $y$ in III is unique by Axiom II. We denote this unique mset $y$ by the symbol $\varnothing$. Hence $\forall x x \notin \varnothing$.

For any mset term $u$ we define the predicate $\operatorname{Set}(u)$ by: $\operatorname{Set}(u)$ stands for $u=\varnothing \vee \forall x \forall n\left(x \in \in^{n} u \rightarrow n=1\right)$. Since $\operatorname{Set}(\varnothing)$, the mset $\varnothing$ is called the empty set. 
We define the msubset relation $\subseteq$ by: for all mset terms $u$ and $v, u \subseteq v$ stands for $\forall z \forall n\left(z \in^{n} u \rightarrow \exists m\left(n \leq m \wedge z \in^{m} v\right)\right)$, in which case we say that $u$ is an msubset of $v$. Clearly $\forall x(\varnothing \subseteq x)$. In relation to its msubsets, an mset is called the parent mset. The relation $\subseteq$ is reflexive and transitive. Using Axioms I and II it is easily shown that $\subseteq$ is also antisymmetric $(\forall x \forall y((x \subseteq y \wedge y \subseteq x) \rightarrow$ $x=y)) . \forall x \forall y((x \subseteq y \wedge \operatorname{Set}(y)) \rightarrow \operatorname{Set}(x))$ also holds.

By defining the msubset relation $\subseteq$ as above, we have rejected the definition given by Meyer and McRobbie [23], p. 128. They define $x \subseteq y$ by $\forall z(z \in$ $x \rightarrow z \in y$ ). Their msubset relation is not antisymmetric and does not preserve the principle that the 'cardinality' of an msubset be at most the 'cardinality' of the parent mset.

We say that $u$ is a part of $v$ if $u \subseteq v \wedge u \neq \varnothing \wedge u \neq v$. We say that $u$ is a whole msubset of $v$ if $\forall z \forall n\left(z \in^{n} u \rightarrow z \in^{n} v\right)$, and that $u$ is a full msubset of $v$ if $u \subseteq v \wedge \forall z(z \in v \rightarrow z \in u)$. A whole msubset 'contains' all multiplicities of common elements, while a full msubset contains every distinct element of the parent mset.

Later we will prove (using the separation schema) that to every mset $x$ there corresponds a unique mset $x^{*}$ (called its root set) such that $x^{*} \subseteq x \wedge \operatorname{Set}\left(x^{*}\right) \wedge$ $\forall z\left(z \in x \rightarrow z \in x^{*}\right)$ - every mset contains a unique full msubset called its root set.

If $u=\varnothing \vee \forall x \forall y((x \in u \wedge y \in u) \rightarrow x=y)$, we say that $u$ is simple. Nonempty simple msets have root sets that contain a single element - all elements of nonempty simple msets are indistinguishable. If $\forall z(z \in u \leftrightarrow z \in v)$, we say that $u$ and $v$ are similar. Similar msets have equal root sets but need not be equal msets. If $\forall x \forall y \forall n \forall m\left(\left(x \in \in^{n} u \wedge y \in \in^{m} u\right) \rightarrow n=m\right)$, then $u$ is called regular (all elements belong with the same multiplicity).

If we restrict Axiom II to 'sets', we obtain

II* $\forall x \forall y((\operatorname{Set}(x) \wedge \operatorname{Set}(y)) \rightarrow(\forall z(z \in x \leftrightarrow z \in y) \rightarrow x=y))$.

This is not the exact analog of the classical axiom of extensionality since elements of 'sets' need not be 'sets' themselves. To obtain exact analogs of sets in MST, we must define 'hereditary sets'.

Let $\operatorname{Trans}(u)$ stand for $\forall x \forall y((x \in y \wedge y \in u) \rightarrow x \in u)$ for any mset term $u$. If $\operatorname{Trans}(u)$ holds, we say that $u$ is transitive. We show (after we discuss the axioms of union, replacement and infinity) that to every mset $x$ there corresponds a unique smallest mset $T C(x)$ (called the transitive closure of $x$ ) such that $\operatorname{Set}(T C(x)) \wedge x^{*} \subseteq T C(x) \wedge \operatorname{Trans}(T C(x))$. The elements of $T C(x)$ are exactly the elements of $x$, the elements of elements of $x$, etc.

We cannot define $\operatorname{Trans}(u)$ by $\forall y(y \in u \rightarrow y \subseteq u)$ in MST. To see this informally, let $[y]_{n}$ be the mset containing exactly $n$ copies of the element $y$. Let $x$ be the mset $\left\{\{y\},[y]_{2},[y]_{3}, \ldots\right\}$; that is, $x$ is the 'set' whose elements are exactly the msets $[y]_{n}$ where $[y]_{1}=\{y\}$. We show that this 'set' exists after the axiom of infinity has been set forth. We would certainly want $\operatorname{Trans}(T C(x))$. Every mset $[y]_{n}$ is an element of $T C(x)$, but it is not possible for every mset $[y]_{n}$ to be an msubset of $T C(x)$. Since $y \in T C(x)$, $m y \in \in^{m} T C(x)$ holds, but then $[y]_{m+1} \nsubseteq T C(x)$.

Let $H \operatorname{Set}(u)$ stand for $\operatorname{Set}(u) \wedge \forall y(y \in T C(u) \rightarrow \operatorname{Set}(y))$. If $H \operatorname{Set}(u)$ holds, then $u$ is called a hereditary set or hset. Clearly, every hset is a set but 
not conversely. In MST the exact analog of the classical axiom of set extensionality is

II** $\quad \forall x \forall y((H \operatorname{Set}(x) \wedge \operatorname{HSet}(y)) \rightarrow(\forall z(z \in x \leftrightarrow z \in y) \rightarrow x=y)$.

There are two elementary mset axioms of MST

IV (i) $\forall x \forall n \exists y\left(x \in^{n} y \wedge \forall z(z \in y \leftrightarrow z=x)\right)$

(ii) $\forall x \forall y\left(x \neq y \rightarrow \forall n \forall m \exists z\left(x \in \in^{n} z \wedge y \in \in^{m} z \wedge\right.\right.$ $\left.\left.\forall z^{\prime}\left(z^{\prime} \in z \leftrightarrow\left(z^{\prime}=x \vee z^{\prime}=y\right)\right)\right)\right)$.

Axiom IV(i) states that for any mset $x$ and any number $n$, there is a unique (by Axiom II) mset $y$ containing exactly $n$ copies of $x$ and nothing else. Let $[u]_{t}$ denote the mset that contains exactly $t$ copies of $u$ and nothing else. We denote $[u]_{1}$ by $\{u\}$ and we call it the singleton set containing $u$ since $\operatorname{Set}\left([u]_{1}\right)$.

Axiom IV(ii) states that for any two distinct msets $x$ and $y$ and any numbers $n$ and $m$, there exists a unique (by Axiom II) mset $z$ containing exactly $n$ copies of $x, m$ copies of $y$, and nothing else. Let $[u, v]_{s, t}$ denote the mset that contains $s$ copies of $u, t$ copies of $v$, and nothing else. We denote $[u, v]_{1,1}$ by $\{u, v\}$ and we call it the pair set containing $u$ and $v$ since $\operatorname{Set}\left([u, v]_{1,1}\right)$.

We require that $x \neq y$ in Axiom IV(ii) since if $x=y$ but $n \neq m$, then IV(ii) would assert the existence of an mset that contradicts Axiom I. We cannot write $\{x, x\}$ in MST since the elements of a pair set must be distinct.

Axiom IV(i) is necessary to ensure the existence of msets whose elements have multiplicities greater than one. Axiom IV(ii), however, is stated as an axiom of MST for convenience. Below we prove (p. 55) IV(ii) as a theorem of MST using Axioms III, IV(i), powerset, replacement, and union.

For mset terms $u$ and $v$ we define

$$
\langle u, v\rangle= \begin{cases}\{\{u\},\{u, v\}\} & \text { if } u \neq v \\ \left\{\{u\},[u]_{2}\right\} & \text { if } u=v\end{cases}
$$

We call $\langle u, v\rangle$ the ordered pair set since $\operatorname{Set}(\langle u, v\rangle)$. Hence, $\langle u, v\rangle$ is always a 'set' of $t w o$ distinct elements (unlike ZF where Kuratowski pairs $\langle x, x\rangle$ are singletons).

For mset terms $u$ and $v$ we define the simple mset $v_{u}$ to be the unique msubset of $v$ that contains all copies of the element $u$ in $v$ (if any) and nothing else. Define $v_{u}=[u]_{t}$ iff $u \in{ }^{t} v$. Therefore, $v_{u}$ is a simple whole msubset of $v$, and distinct $u$ 's in $v$ give rise to distinct $v_{u}$ 's. Using Axiom II, we have $\forall x \forall y\left(y_{x}=\varnothing \leftrightarrow x \notin y\right)$ and $\forall x \forall y \forall n\left(y_{x}=[x]_{n} \leftrightarrow x \in^{n} y\right)$.

For simple msets of the form $[u]_{t}$ we define the numeric term $\left|[u]_{t}\right|$ as follows: $\left|[u]_{t}\right|=t$ for any mset term $u$. Therefore, for any mset terms $u$ and $v$ and any numeric term $t,\left|v_{u}\right|=t$ iff $u \in^{t} v$ and $\left|v_{u}\right|$ is unique by Axiom I. For simple msets of the form $[u]_{t}$ we also define the cardinality of $[u]_{t}$, denoted by $C\left([u]_{t}\right)$, to be $\hat{t}$. The cardinality of a simple mset is an mset (in fact, a hereditary set). For any mset terms $u$ and $v$ and any numeric term $t, C\left(v_{u}\right)=\hat{t}$ iff $u \in \in^{t} v$ and $C\left(v_{u}\right)$ is unique by Axiom I.

The powerset axiom of MST is

V $\forall x \exists y(\operatorname{Set}(y) \wedge \forall z(z \in y \leftrightarrow z \subseteq x))$. 
In other words, for every mset $x$ there is a set whose elements are exactly the msubsets of $x$. The set $y$ whose existence is postulated in Axiom $\mathrm{V}$ is unique by Axiom II*. For any mset term $u$, we denote the set of all msubsets of $u$ by $\mathbb{P}(u)$, and we call it the powerset of $u$. For example,

$$
\mathbb{P}\left([x, y]_{3,1}\right)=\left\{\varnothing,\{x\},[x]_{2},[x]_{3},\{y\},\{x, y\},[x, y]_{2,1},[x, y]_{3,1}\right\} .
$$

Informally, if $x$ is a 'set' with $n$ distinct elements, then $\mathbb{P}(x)$ contains exactly $2^{n}$ distinct elements. If $x$ is a nonset with $n$ elements (repetitions counted), then $\mathbb{P}(x)$ contains strictly less than $2^{n}$ elements because singleton msubsets do not repeat in $\mathbb{P}(x)$ since $\operatorname{Set}(\mathbb{P}(x)) . \forall x \mathbb{P}(x) \neq \varnothing$ also, since $\forall x \varnothing \in \mathbb{P}(x)$.

Our formal definition of $\mathbb{P}(x)$ is equivalent to the informal definition in Hickman ([15], p. 213). Why require that $\mathbb{P}(x)$ be a set? Hickman answers: “. . . we could see no good reason for introducing repeated elements into a powerset. However . . . this does mean that one of the cornerstones of classical set theory, Cantor's powerset theorem, fails for multisets" ([15], p. 213).

In fact, it is possible to formulate a reasonable definition of a power $\mathrm{mul}$ tiset of $x$ for finite msets $x$ which preserves Cantor's theorem. Conceptually, we think of $x$ as if it were a set (we think of the elements of $x$ as if they were all distinct). We take the classical powerset of $x$ and then undo the 'distinctions' made in the first step. The result (with repetitions counted) is the power multiset $\tilde{\mathbb{P}}(x)$. We define $\tilde{\mathbb{P}}(x)$ informally as follows: $(\tilde{\mathbb{P}}(x))^{*}=\tilde{\mathbb{P}}(x)$ (so $y \in \tilde{\mathbb{P}}(x) \leftrightarrow$ $y \subseteq x)$. If $y=\varnothing$, then $y \in^{1} \tilde{\mathbb{P}}(x)$; and if $y \neq \varnothing$, then $y \in^{k} \tilde{\mathbb{P}}(x)$ where

$$
k=\prod_{z \in y^{*}}\left(\begin{array}{l}
\left|x_{z}\right| \\
\left|y_{z}\right|
\end{array}\right) \text { and }\left(\begin{array}{c}
n \\
m
\end{array}\right)=\frac{n !}{m !(n-m) !}
$$

is the number of combinations of $n$ objects taken $m$ at a time, and where the product $\prod_{z \in y^{*}}$ is taken over distinct elements $z$ of the msubset $y$ and where $\left|x_{z}\right|=n$ iff $z \epsilon^{n} x$ and $\left|y_{z}\right|=m$ iff $z \in^{m} y$. For example, if $x=[\varnothing,\{\varnothing\}]_{3,1}$ and $y=[\varnothing]_{2}$, then $k=\left(\begin{array}{l}3 \\ 2\end{array}\right)=3$. With the same mset $x$, if $y=[\varnothing,\{\varnothing\}]_{2,1}$ then $k=\left(\begin{array}{l}3 \\ 2\end{array}\right)\left(\begin{array}{l}1 \\ 1\end{array}\right)=3$. If the mset $x$ contains $n$ elements, then $\tilde{\mathbb{P}}(x)$ contains exactly $2^{n}$ elements. We do not use $\tilde{\mathbb{P}}(x)$ in MST because for certain infinite msets the combinatorial formula generates infinite multiplicities. For example, if $x=\left[x_{1}, x_{2}, x_{3}, \ldots\right]_{2,2,2, \ldots}$ and $y=\left[x_{1}, x_{2}, x_{3}, \ldots\right]_{1,1,1, \ldots}=\left\{x_{1}, x_{2}, x_{3}, \ldots\right\}$, then $y \subseteq x$ and $y \in \in^{k} \tilde{\mathbb{P}}(x)$ where $k=\left(\begin{array}{l}2 \\ 1\end{array}\right)\left(\begin{array}{l}2 \\ 1\end{array}\right)\left(\begin{array}{l}2 \\ 1\end{array}\right) \ldots$ To avoid such difficulties we require that $\forall x \operatorname{Set}(\mathbb{P}(x))$ as in Axiom V. Before discussing Cantor's powerset theorem for msets, we introduce functions between msets.

Multisets that are functions (as defined below) are denoted by symbols $f, g, h, \ldots$ We define functions between msets by first defining functions between root sets. The definition of a function between root sets is just the classical definition of function. For any mset terms $u$ and $v$, the mset $f$ is a function from $u^{*}$ to $v^{*}$ (written $f: u^{*} \rightarrow v^{*}$ ) iff

(i) $\operatorname{Set}(f) \wedge$

(ii) $\forall z\left(z \in f \rightarrow \exists z_{1} \exists z_{2}\left(z_{1} \in u^{*} \wedge z_{2} \in v^{*} \wedge z=\left\langle z_{1}, z_{2}\right\rangle\right)\right) \wedge$ 
(iii) $\forall z_{1}\left(z_{1} \in u^{*} \rightarrow \exists z_{2}\left(z_{2} \in v^{*} \wedge\left\langle z_{1}, z_{2}\right\rangle \in f\right)\right) \wedge$

(iv) $\forall z_{1} \forall z_{2} \forall z_{2}^{\prime}\left(\left(\left\langle z_{1}, z_{2}\right\rangle \in f \wedge\left\langle z_{1}, z_{2}^{\prime}\right\rangle \in f\right) \rightarrow z_{2}=z_{2}^{\prime}\right)$.

The function $f: u^{*} \rightarrow v^{*}$ is an injection iff

(v) $\forall z_{1} \forall z_{1}^{\prime} \forall z_{2}\left(\left(\left\langle z_{1}, z_{2}\right\rangle \in f \wedge\left\langle z_{1}^{\prime}, z_{2}\right\rangle \in f\right) \rightarrow z_{1}=z_{1}^{\prime}\right)$.

The function $f: u^{*} \rightarrow v^{*}$ is a surjection iff

(vi) $\forall z_{2}\left(z_{2} \in v^{*} \rightarrow \exists z_{1}\left(z_{1} \in u^{*} \wedge\left\langle z_{1}, z_{2}\right\rangle \in f\right)\right)$.

The function $f: u^{*} \rightarrow v^{*}$ is a bijection iff (v) and (vi) hold.

For functions between arbitrary msets we require that images of indistinguishable elements of the domain must be indistinguishable elements of the range, but images of distinct elements of the domain need not be distinct elements of the range.

A function $f$ from mset $x$ to mset $y$ is 'rooted' in the function $f$ from $x^{*}$ to $y^{*}$. We think of $x$ as partitioned into whole simple disjoint msubsets $x_{z}$, and $y$ as partitioned into whole simple disjoint msubsets $y_{z^{\prime}}$. Depending upon the nature of the 'root' function $f$ from $x^{*}$ to $y^{*}$ certain msubsets $y_{z^{\prime}}$ will be of the form $y_{f(z)}$ where $z \in x$. We think of the function $f$ from $x$ to $y$ as 'acting upon' all copies of $z$ in $x_{z}$ and some copies of $f(z)$ in $y_{f(z)}$ (although which copies of $f(z)$ in $y_{f(z)}$ cannot be determined). Therefore, all the elements of the msubset $x_{z} \subseteq x$ are mapped by $f$ to some msubset of $y_{f(z)}$ in $y$. We now give the formal definitions.

Let $u$ and $v$ be msets. A function from $u$ to $v$ is defined to be a function from $u^{*}$ to $v^{*}$. Therefore every function between msets is a set of ordered pair sets. Let $f: u \rightarrow v$ mean that $f$ is a function from $u$ to $v$ (we think of $f$ as a function between multisets).

The function $f: u \rightarrow v$ is an injection iff

(i) $f: u^{*} \rightarrow v^{*}$ is an injection and

(ii) $\forall z\left(z \in u^{*} \rightarrow\left|u_{z}\right| \leq\left|v_{f(z)}\right|\right)$.

The function $f: u \rightarrow v$ is a surjection iff

(i) $f: u^{*} \rightarrow v^{*}$ is a surjection and

(ii) $\forall z\left(z \in u^{*} \rightarrow\left|u_{z}\right| \geq\left|v_{f(z)}\right|\right)$.

The function $f: u \rightarrow v$ is a bijection iff it is an injection and a surjection; that is, iff

(i) $f: u^{*} \rightarrow v^{*}$ is a bijection, and

(ii) $\forall z\left(z \in u^{*} \rightarrow\left|u_{z}\right|=\left|v_{f(z)}\right|\right)$.

These definitions can be shown to be equivalent to those in [15], p. 213.

If $x \subseteq y$, then the identity map $f: x^{*} \rightarrow x^{*} \subseteq y^{*}$ is a function $f: x \rightarrow y$ which is an injection (the natural embedding) of $x$ into $y$. Given $f:\{x\} \rightarrow\{y\}$ where $f=\{\langle x, y\rangle\}$, the function $f:[x]_{2} \rightarrow[y]_{100}$ is an injection. Since copies of $y$ in $[y]_{100}$ are indistinguishable, we cannot determine which copies of $y$ are images of $x$ and which copies are not images of $x$. The function $f:[x]_{10} \rightarrow[y]_{9}$ is a surjection. One copy of $y$ is the image of more than one copy of $x$, but since all copies of $y$ are indistinguishable, we cannot determine the copy of $y$ for which 
this is the case. If $f:\{x, y\} \rightarrow\left\{z, z^{\prime}\right\}$ where $f=\left\{\langle x, z\rangle,\left\langle y, z^{\prime}\right\rangle\right\}$ then $f$, as a function between sets, is a bijection. However, $f:[x, y]_{10,2} \rightarrow\left[z, z^{\prime}\right]_{5,5}$ is neither an injection nor a surjection. Given the surjection $f:[x]_{2} \rightarrow[z]_{2}$, if we 'increase' the domain to $[x, y]_{2,1}$ then the function $g:[x, y]_{2,1} \rightarrow[z]_{2}$ is no longer a surjection.

Msets of equal 'cardinality' need not have a bijection between them. The msets $[x, y]_{2,1}$ and $\{x, y, z\}$ both contain three elements, but there can be no bijection between them since multiplicities in the domain do not equal multiplicities in the range.

For mset terms $u$ and $v$, we define the binary relations,

$u \leq v$ stands for "there is an injection $f: u \rightarrow v$ "

$u \approx v$ stands for "there is a bijection $f: u \rightarrow v$ "

$u<v$ stands for $u \leq v \wedge \sim u \approx v$.

Hickman ([15], p. 215) shows that Cantor's theorem $(\forall x x<\mathbb{P}(x))$ fails for multisets. If $\sim \operatorname{Set}(x)$ then $\exists y \exists n\left(y \in^{n} x \wedge n>1\right)$. For any function $f: x \rightarrow \mathbb{P}(x)$ it must be that $\left|x_{y}\right|>\left|\mathbb{P}(x)_{f(y)}\right|=1$ since $\operatorname{Set}(\mathbb{P}(x))$. Hence there is no injection from $x$ into $\mathbb{P}(x)$ when $\sim \operatorname{Set}(x)$. However, Cantor's theorem is provable in MST for all sets $x$. The proof is identical to the classical proof ([14], p. 93).

Cantor's theorem is provable in MST for finite msets $x$ using the power multiset $\tilde{\mathbb{P}}(x)$. Define the function $f: x^{*} \rightarrow(\tilde{\mathbb{P}}(x))^{*}$ by $f(y)=\{y\} \subseteq x$ for each $y \in x^{*}$. The function $f$ between sets is an injection. The function $f: x \rightarrow \widetilde{\mathbb{P}}(x)$ between msets is also an injection since for all $y \in x^{*}$, if $y \in^{n} x$ then $f(y)=$ $\{y\} \in^{k} \tilde{\mathbb{P}}(x)$ where $k=\left(\begin{array}{c}|x y| \\ \left|\{y\}_{y}\right|\end{array}\right)=\left(\begin{array}{c}n \\ 1\end{array}\right)=n$. Therefore, if $y \in^{n} x$ then $f(y) \in^{n} \tilde{\mathbb{P}}(x)$, for all $y \in x^{*}$. Hence, $x \leq \tilde{\mathbb{P}}(x)$. Assuming that $x \approx \tilde{\mathbb{P}}(x)$ gives a bijection $g: x^{*} \rightarrow(\tilde{\mathbb{P}}(x))^{*}=\mathbb{P}(x)$, which can be used (exactly as in the classical proof) to generate a contradiction. Therefore, for all finite msets $x, x<$ $\tilde{\mathbb{P}}(x)$.

Since every bijection is an injection and $\approx$ commutes, $\forall x \forall y(x \approx y \rightarrow(x \leq$ $y \wedge y \leq x)$ ). However, Hickman ([15], p. 215) shows that the Schröder-Bernstein Theorem $\forall x \forall y((x \leq y \wedge y \leq x) \rightarrow x \approx y)$ fails for multisets, using the following example:

$$
\begin{aligned}
& \text { let } x=\left[x_{1}, x_{2}, x_{3}, \ldots\right]_{2,4,6, \ldots} \text { and } \\
& \text { let } y=\left[y_{0}, y_{1}, y_{2}, y_{3}, \ldots\right]_{1,3,5,7, \ldots} \text {. }
\end{aligned}
$$

The function $f: x^{*} \rightarrow y^{*}$ defined by $f\left(x_{n}\right)=y_{n}$ makes $f: x \rightarrow y$ an injection so that $x \leq y$. The function $g: y^{*} \rightarrow x^{*}$ defined by $g\left(y_{n}\right)=x_{n+1}$ makes $g: y \rightarrow x$ an injection so that $y \leq x$. There cannot be a bijection $h: x \rightarrow y$ since all multiplicities in $x$ are even and all multiplicities in $y$ are odd. Therefore, $x \neq y$.

The axiom of foundation of MST is

$$
\text { VI } \forall y(y \neq \varnothing \rightarrow \exists x(x \in y \wedge \forall z(z \in x \rightarrow z \notin y))) \text {. }
$$

It states that every nonempty mset contains an ' $\in$-minimal element' (an element from which it is disjoint). Thus our defined binary membership relation $\in$ is well-founded. Axiom VI disallows infinitely descending $\epsilon$-chains within an mset of the form $\ldots \in x_{3} \in x_{2} \in x_{1}$ since the msubset whose elements are the elements of the chain would have no $\in$-minimal element. Therefore, 'extraordi- 
nary' msets $x$ such that $x=\{x\}$ and $\in$-loops of the form $x_{1} \in x_{2} \in \ldots \in x_{n}=$ $x_{1}$ are ruled out in MST. It follows that $\forall x x \notin x$, or $\forall x x_{x}=\varnothing$, or msets $x$ and $\{x\}$ are disjoint. $\forall x \forall y(x \in y \rightarrow y \notin x)$ also holds, or in other notation, $\forall x \forall y\left(y_{x} \neq \varnothing \rightarrow x_{y}=\varnothing\right)$.

The union axiom of MST is

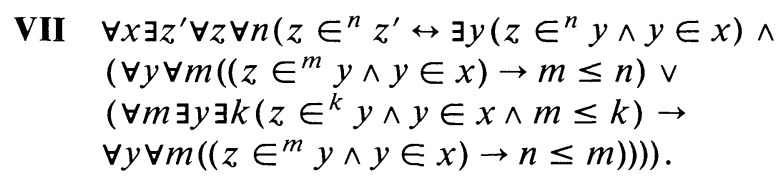

In words, for any mset $x$, there is an mset $z^{\prime}$ which consists of all elements of elements of $x$. The multiplicity $n$ of $z$ in $z^{\prime}$ equals the maximum multiplicity of $z$ as an element of elements of $x$, if such a maximum exists. If the multiplicities of $z$ as an element of elements of $x$ are not bounded above, the multiplicity $n$ of $z$ in $z^{\prime}$ equals the minimum multiplicity of $z$ as an element of elements of $x$. Such a minimum multiplicity always exists by the well-ordering $\leq$ in PA.

The mset $z^{\prime}$ in Axiom VII is unique by Axiom II and is called the union of $x$. For any mset term $v$ let $\cup v$ denote the union of $v$. For mset terms $u$ and $v$, we define the binary mset union operation $U$ by:

$u \cup v=\cup\{u, v\}$ if $u \neq v$ and

$u \cup u=\cup\{u\}$ otherwise.

By Axiom II, we have $\forall x(x \cup x=x)$.

For all numeric terms $s$ and $t$, we define a binary function $\max$ as follows:

$$
\max (s, t)= \begin{cases}t & \text { if } s \leq t \\ s & \text { otherwise }\end{cases}
$$

which is well-defined by the law of trichotomy in PA.

Our formal mset $x \cup y$ has the same properties as found in the literature ([15], [17], [22], and [23]); that is,

$$
\begin{aligned}
& \forall x \forall y \forall z \forall n\left(z \in ^ { n } x \cup y \leftrightarrow \left[\left(z \in^{n} x \wedge z \notin y\right) \vee\right.\right. \\
& \left.\left.\left(z \notin x \wedge z \in^{n} y\right) \vee \exists k \exists l\left(z \in^{k} x \wedge z \in^{l} y \wedge n=\max (k, l)\right)\right]\right) .
\end{aligned}
$$

For finite msets $x$, the maximum multiplicities of elements of elements of $x$ always exist. For certain infinite msets like $x=\left\{\{y\},[y]_{2},[y]_{3}, \ldots\right\}$ the maximum multiplicity of elements of elements of $x$ does not exist; thus $\cup x=\{y\}$, that is, the minimum multiplicity is used instead. Let $x \neq \varnothing$. The multiplicity of any $y \in x$ is irrelevant in $\cup x$. Hence, $\cup x=\cup x^{*}$. The unions of similar msets are equal; that is, all msets with the same root have the same union$\forall x \forall y\left(x^{*}=y^{*} \rightarrow \cup x=\cup y\right)$. The converse is false since $\cup\{\varnothing\}=\cup \varnothing=\varnothing$ but $\{\varnothing\}^{*}=\{\varnothing\} \neq \varnothing^{*}=\varnothing$. In MST we have

$$
\begin{aligned}
& \forall x(\cup x=\varnothing \leftrightarrow x=\varnothing \vee \forall y(y \in x \rightarrow y=\varnothing)) \text { or } \\
& \forall x\left(\cup x=\varnothing \leftrightarrow x=\varnothing \vee \exists n x=[\varnothing]_{n}\right) .
\end{aligned}
$$

For $y \neq \varnothing, y \cup \varnothing=\cup\{y, \varnothing\}=y$. Also, in general, $(\cup x)^{*} \neq \cup x^{*}$ since if $(\cup x)^{*}=\cup x^{*}=\cup x$ then $\forall x \operatorname{Set}(\cup x)$, which is nonsense. If $\operatorname{Set}(x)$, then $\cup x$ need not be a set. For example, if $x=\left\{[y]_{2}\right\}$ then $\operatorname{Set}(x)$ but $\sim \operatorname{Set}(\cup x)$ since 
$\cup x=[y]_{2}$. However, if every element of $x$ is a set, then $\cup x$ is a set independent of $x$ itself. If $H \operatorname{Set}(x)$ then $H \operatorname{Set}(\cup x)$ and $\cup x$ is equivalent to classical set union. We have chosen to define $U x$ to include all elements of elements of $x$ (with maximum multiplicity when the maximum exists, and minimum multiplicity otherwise) in order to preserve the desired relation $\cap x \subseteq \cup x \subseteq \uplus x$, which will be proved shortly. However, one consequence of this approach is that there are msets $x$ and $y$ such that $x \subseteq y$ but $\cup x \nsubseteq \cup y$. If, for example, $x=\left\{[z]_{2}\right\}$ and $y=\left\{\{z\},[z]_{2},[z]_{3}, \ldots\right\}$, then $x \subseteq y$ but $\cup x=[z]_{2} \nsubseteq \cup y=\{z\}$.

We now define the von Neumann numerals in MST as follows: for any mset term $u$,

$$
\begin{array}{ll}
\text { Conn }(u) \text { stands for } & \forall y \forall z((y \in u \wedge z \in u) \rightarrow(y \in z \vee z \in y \vee y=z)) ; \\
\operatorname{Lim}(u) \text { stands for } & \operatorname{Trans}(u) \wedge \operatorname{Conn}(u) \wedge u \neq \varnothing \wedge \forall y(y \in u \rightarrow \\
& y \cup\{y\} \in u) ; \text { and } \\
V N N(u) \text { stands for } & H \operatorname{Set}(u) \wedge \operatorname{Trans}(u) \wedge \operatorname{Conn}(u) \wedge \sim \operatorname{Lim}(u) \wedge \\
& \forall y(y \in u \rightarrow \sim \operatorname{Lim}(y)) .
\end{array}
$$

For example, the set $\mathbb{P}(\{\varnothing,\{\varnothing\}\})=\{\varnothing,\{\varnothing\},\{\{\varnothing\}\},\{\varnothing,\{\varnothing\}\}\}$ is transitive but not connected (pick $\varnothing$ and $\{\{\varnothing\}\})$. We require $\operatorname{HSt}(u)$ in the definition of $V N N(u)$ to exclude msets like $[\varnothing]_{2}$ and $\left\{\varnothing,[\varnothing]_{2},\left\{\varnothing,[\varnothing]_{2}\right\}\right\}$. Using the axiom of infinity we show later that the class term $\{u \mid V N N(u)\}$ is a set.

The numeric-mset correspondence axiom is

N8 $\hat{0}=\varnothing \wedge \widehat{\mathrm{S} 0}=\{\varnothing\} \wedge \forall n(\widehat{\mathrm{S} n}=\hat{n} \cup\{\hat{n}\})$.

$V N N(\hat{n})$ is a wff of $L$ and it is straightforward to show that $V N N(\hat{0}) \wedge$ $\forall \dot{n}(V N N(\hat{n}) \rightarrow V N N(\widehat{S} \dot{n}))$ holds. By the induction schema, we have $\forall \dot{n} V N N(\hat{n})$. Using the separation schema, we also show later that $\forall x(V N N(x) \rightarrow \exists \dot{m} x=\hat{\dot{m}})$ holds. The numbers of PA are mapped by " to their mset associates (the von Neumann numerals) and this association is a 1-1 correspondence. We use the correspondence in the next axiom to state formally when a class term is and is not finite. We will also need the correspondence to define the cardinality of msets (it was Robin Gandy who observed that the correspondence is, in fact, necessary in MST).

The additive union axiom of MST is

VIII $\forall x \exists z^{\prime} \forall z \forall n\left(z \in^{n} z^{\prime} \leftrightarrow z \in \cup x \wedge\right.$

$$
\begin{aligned}
& \exists m\left(\exists f f: \hat{m} \frac{1-1}{\text { onto }}\left\{y \mid y \in x^{*} \wedge z \in y\right\} \rightarrow\right. \\
& \left(\exists k \exists l \left(\forall i\left(i<m \rightarrow\left(f_{i} \in(k)_{l} x \wedge z \in \in^{(l)_{l}} f_{i}\right)\right) \wedge\right.\right. \\
& \left.\left.\left.\left.n=\sum_{i<m}(k)_{i} \cdot(l)_{i}\right)\right)\right) \wedge\left(\sim \exists m \exists f f: \hat{m} \frac{1-1}{\text { onto }}\left\{y \mid y \in x^{*} \wedge z \in y\right\} \rightarrow z \in \in^{n} \cup x\right)\right)
\end{aligned}
$$

where $f: \hat{m} \frac{1-1}{\text { onto }}\left\{y \mid y \in x^{*} \wedge z \in y\right\}$ stands for the formal statement in $L$ " $f$ is a bijection from the mset $\hat{m}$ onto the mset equal to the class term $\left\{y \mid y \in x^{*} \wedge\right.$ $z \in y\} \subseteq x^{*}$, where $f_{i}$ is the image of $\hat{i} \in \hat{m}$ under $f$ (that is, $f_{i}=f(\hat{i})$ is some $y$ in $x^{*}$ containing the element $z$ ), where $k$ (or $l$ ) equals the numeric term that codes the finite sequence $\left\langle(k)_{l}\right\rangle$ (or $\left.\left\langle(l)_{i}\right\rangle\right)$ of length $m$ where $(k)_{i}$ is the multiplicity of $f_{i}$ in $x$ (or $(l)_{i}$ is the multiplicity of $z$ in $f_{l}$ ), and where the expression $n=\sum_{i<m}(k)_{i} \cdot(l)_{l}$ is formally expressible as a wff in the language of PA.

It is well-known that Gödel's $\beta$-function is formally expressible in the language of PA, and that it can be made to behave like any finite sequence in PA 
and, therefore, like any finite sum in PA - a finite sequence of partial sums (see, for example, [9], pp. 11, 114).

In words, Axiom VIII states that, for every mset $x$, there is a unique (by Axiom II) mset $z^{\prime}$ which contains all elements $z$ of elements $y$ of $x$. Thus, the mset $z^{\prime}$ has exactly the same elements as the mset $\cup x$. The multiplicity $n$ of each such $z$ in $z^{\prime}$ is determined as follows:

1. if $z$ belongs to at most a finite number of elements $y$ of $x$, then $n=$ $\sum_{y}\left|x_{y}\right| \cdot\left|y_{z}\right|$ where the finite sum $\sum_{y}$ is taken over the finite number of msets $y$ in $x^{*}$ that contain $z$ as an element; and

2. if $z$ belongs to infinitely many elements $y$ of $x$, then $n$ equals the multiplicity of $z$ in $\cup x$. The net effect is that the multiplicity $n$ of $z$ in $z^{\prime}$ equals:

(i) the sum of the products of the multiplicity of $z$ as an element of elements $y$ of $x$ times the multiplicity of that same $y$ in $x$, when this sum is finite, or

(ii) the maximum of the multiplicities of $z$ as an element of elements of $x$, when this maximum is finite, or

(iii) the minimum of the multiplicities of $z$ as an element of elements of $x$, which is always finite.

The mset $z^{\prime}$ in Axiom VIII is called the additive union of $x$. For any mset term $v$, we denote the additive union of $v$ by $\uplus v$. For mset terms $u$ and $v$, we define the binary additive union operation $\uplus$ as follows:

$$
u \uplus v= \begin{cases}\uplus\{u, v\} & \text { if } u \neq v \\ \uplus[u]_{2} & \text { if } u=v .\end{cases}
$$

If $x$ is finite, then the sum of products of multiplicities of elements of elements of $x$ is always finite. The mset $x \uplus y$ has the following property:

$$
\begin{aligned}
& \forall x \forall y \forall z \forall n\left(z \in ^ { n } x \uplus y \leftrightarrow \left[\left(z \in^{n} x \wedge z \notin y\right) \vee\right.\right. \\
& \left.\left.\left(z \notin x \wedge z \in^{n} y\right) \vee \exists k \exists l\left(z \in^{k} x \wedge z \in^{l} y \wedge n=k+l\right)\right]\right) .
\end{aligned}
$$

The binary operation $\uplus$ was first introduced by Knuth [17] but corresponds exactly with Weierstrass's "sum" in [4], pp. 19-20, Hailperin's heap sum " + " in [12], p. 89, and Meyer and McRobbie's product “." in [23], p. 128. Hickman [15] does not introduce the $\uplus$ operation. He states incorrectly ([15], p. 214) that Knuth replaces $\cup$ by $\uplus$ when, in fact, Knuth uses both operations. This may in part explain why Hickman sees "no logical reason" for introducing the $\uplus$ operation between msets.

It should be noted that the additive union Axiom VIII is not independent: it is provable using the other axioms of MST (most notably, the Peano, union, and replacement axioms). We will not give the proof.

If $x$ is an infinite mset such that some mset $z$ belongs to an infinite number of elements of $x$, then the sum of products for $z$ as an element of elements of $x$ is not finite, and cannot therefore serve as the multiplicity of $z$ in $\uplus x$. If 
this is the case, we use the multiplicity of $z$ in $\cup x$ instead. If $x=\left\{\{z\},[z]_{2}\right.$, $\left.[z]_{3}, \ldots\right\}$, both the sum of products and the maximum of multiplicities of $z$ as an element of elements of $x$ do not exist. Thus $\uplus x=\cup x=\{z\}$.

If $x \neq \varnothing$ the multiplicities of msets $y \in x$ are relevant to $\uplus x$, and so, in general, $\uplus x \neq \uplus x^{*}$. Generally $(\uplus x)^{*} \neq \uplus x^{*}$, since $(\uplus x)^{*}$ is always a set, but $\uplus x^{*}$ need not be a set. Clearly, $\uplus \varnothing=\uplus\{\varnothing\}=\varnothing$. Since $\forall z \forall n\left(z \in^{n} y \leftrightarrow z \in^{2 n}\right.$ $y \uplus y)$ and $\forall n(n \neq 2 \cdot n)$, we have that if $y \neq \varnothing$, then $y \uplus y=\uplus[y]_{2} \neq y$. Also, $y \uplus \varnothing=y$ since $\forall n(n+0=n)$.

Since $\cup x$ and $\uplus x$ contain all elements of elements of $x,(\cup x)^{*}=(\uplus x)^{*}$. Also, $\cup x \subseteq \uplus x$ since if $z \in^{k} \cup x$ then either $z \in^{k} \uplus x$ or $z \in{ }^{n} \uplus x$, where $n \geq$ $n_{1}+n_{2}+\ldots+n_{m} \geq \max \left\{n_{1}, n_{2}, \ldots, n_{m}\right\} \geq \min \left\{n_{1}, n_{2}, \ldots, n_{m}\right\}$. Hence, $k \leq n$.

The axiom schema of separation of MST states that for every wff $\varphi(x, n)$ of $L$ with free variables including $x$ and $n$ but excluding $y$ and $n^{\prime}$, the universal closure of

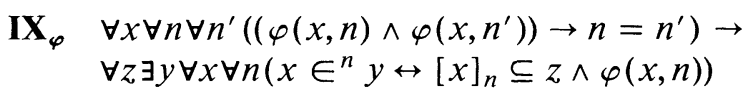

is an axiom of MST.

We require that $\varphi(x, n)$ be 'functional' since if there is more than one $n$ for some $x \in z$ then the mset $y$ is not well-defined. In all such axioms $\mathrm{IX}_{\varphi}$, we have $y \subseteq z$ since $\forall x \forall n\left(x \in \in^{n} y \rightarrow[x]_{n} \subseteq z\right)$.

The 'limitation of size' principle is the requirement that $[x]_{n} \subseteq z$ : the multiplicity of each $x$ in $y$ is no greater than its multiplicity in the given mset $z$. Msets that exist by the separation schema are always msubsets of already existing msets.

If the wff $\varphi(x, n)$ is $x \in^{n} z$ then $\varphi(x, n)$ is 'functional' by Axiom I and $y=z$ in $\operatorname{IX}_{\varphi}$. If $\varphi(x, n)$ is $x \neq x \wedge n=1$ then $y=\varnothing \subseteq z$ in $\operatorname{IX}_{\varphi}$. If $\varphi(x, n)$ is $x=z^{\prime} \wedge x \in \in^{n} z$ then $y=z_{z^{\prime}}$ in Axiom IX $\mathrm{I}_{\varphi}$; that is, $y$ is the simple whole msubset $z_{z^{\prime}} \subseteq z$ that contains all copies of $z^{\prime}$ in $z$ if any. If $z^{\prime} \notin z$ then $z_{z^{\prime}}=\varnothing \subseteq z$.

We can now prove $\forall x(V N N(x) \rightarrow \exists \dot{m} x=\hat{\dot{m}})$. Suppose $\exists y(V N N(y) \wedge$ $\sim \exists \dot{m} y=\hat{\dot{m}}$ ) holds. Since $\{x \mid V N N(x)\}$ is a set and since $\sim \exists \dot{m} m x=\hat{m} \wedge n=1$ is a wff $\varphi(x, n)$ with free variables $x$ and $n$ (but not $y$ and $n^{\prime}$ ), the separation schema gives us that $\{x \mid V N N(x) \wedge \sim \exists \dot{m} x=\hat{m} \wedge n=1\}$ is a set. It is nonempty by our assumption, so by Axiom VI it contains an $\in$-least element $z$. Now $z \neq \varnothing$ since $\hat{0}=\varnothing$ by N8. Therefore $z=y \cup\{y\}$ for some $y$ such that $V N N(y)$ and $y=\hat{n}$. But then $z=y \cup\{y\}=\hat{n} \cup\{\hat{n}\}=\hat{S} n$ by N8 which contradicts $\sim \exists \dot{m} z=$ $\hat{\dot{m}}$. We proved earlier that $\forall \dot{n} V N N(\hat{\hat{n}})$ holds. Therefore, the range of the correspondence is exactly the set of von Neumann numerals; that is, $\forall x(\exists \dot{n} x=$ $\hat{n} \leftrightarrow V N N(x))$.

We now use the separation axioms $\mathrm{IX}_{\varphi}$ to prove the existence of root sets, intersection msets and relative complement msets. If the wff $\varphi(x, n)$ is $x=x \wedge$ $n=1$ then the consequent of Axiom $\operatorname{IX}_{\varphi}$ is

$$
\forall z \exists y \forall x \forall n\left(x \in \in^{n} y \leftrightarrow(\{x\} \subseteq z \wedge n=1)\right) .
$$

The mset $y$ in $\operatorname{IX}_{\varphi}$ is the root set $z^{*}$ of $z$ such that $\forall z \operatorname{Set}\left(z^{*}\right)$. Therefore, every mset $z$ has a unique full msubset $z^{*}$. Clearly $\varnothing^{*}=\varnothing$. Theorems of MST include 


$$
\begin{aligned}
& \forall x\left(x^{*}\right)^{*}=x^{*}, \forall x\left(\operatorname{Set}(x) \leftrightarrow x=x^{*}\right), \\
& \forall x \forall y\left(x \in y \leftrightarrow x \in y^{*}\right), \text { and } \forall x \forall y\left(x \subseteq y \rightarrow x^{*} \subseteq y^{*}\right)
\end{aligned}
$$

The converse of the last sentence is false since, for example, $[\varnothing]_{n+1} \nsubseteq[\varnothing]_{n}$.

If the mset $z$ in $\operatorname{IX}_{\varphi}$ is $\cup z$ and the wff $\varphi(x, n)$ is $\exists z^{\prime}\left(x \in{ }^{n} z^{\prime} \wedge z^{\prime} \in z\right) \wedge$ $\forall z^{\prime}\left(z^{\prime} \in z \rightarrow \exists m\left(x \in \in^{m} z^{\prime} \wedge n \leq m\right)\right)$, then the unique mset $y$ in $\operatorname{IX}_{\varphi}$ is called the intersection of $z$. For any mset term $v$, the intersection of $v$ is denoted by $\cap v$. The separation schema gives immediately $\cap x \subseteq \cup x$, and we have the desired relation $\cap x \subseteq \cup x \subseteq \uplus x$.

In general, $\cap x$ is the unique mset that contains all elements of elements of $x$ that belong to every element of $x$. The multiplicity of an element in $\cap x$ is the minimum multiplicity of that element as an element of every element of $x$. The minimum multiplicity always exists since $\leq$ is a well-order in PA.

For any mset terms $u$ and $v$, we define the binary intersection operation $\cap$ as follows:

$$
u \cap v= \begin{cases}\cap\{u, v\} & \text { if } u \neq v \\ \cap\{u\} & \text { if } u=v\end{cases}
$$

If $u \cap v=\varnothing$ we say that $u$ and $v$ are disjoint.

For all numeric terms $s$ and $t$, we define the binary function min as follows:

$$
\min (s, t)= \begin{cases}s & \text { if } s \leq t \\ t & \text { otherwise }\end{cases}
$$

The function min is well-defined by the law of trichotomy in PA.

The mset $x \cap y$ has the properties of mset intersection found in the literature ([15], [17], [22], and [23]), namely,

$$
\forall x \forall y \forall z \forall n\left(z \in^{n} x \cap y \leftrightarrow \exists k \exists l\left(z \in^{k} x \wedge z \in^{l} y \wedge n=\min (k, l)\right)\right) .
$$

Let $x \neq \varnothing$ be some mset. For any $y \in x, y \cap y=\cap\{y\}=y$ by Axiom II. Hence, the multiplicities of elements $y \in x$ are irrelevant to the definition of $\cap x$. Hence, $\cap x=\cap x^{*}$. In general, $(\cap x)^{*} \neq \cap x^{*}$ since $\cap x^{*}$ is not always a set but $\forall x \operatorname{Set}\left((\cap x)^{*}\right)$. For any $y \in x$ where $y \neq \varnothing, y \cap \varnothing=\cap\{y, \varnothing\}=\varnothing$. If $\varnothing \in x$, then $\cap x=\varnothing$. If elements of $x$ are pairwise disjoint, or if a single element of $x$ is disjoint from any other element of $x$, then $\cap x=\varnothing$. Also, $\cap \varnothing=\cap\{\varnothing\}=\varnothing$. If $x=\left\{\{z\},[z]_{2},[z]_{3}, \ldots\right\}$ then $\cap x=\{z\}$.

It is easy to prove $\forall x \forall y(x \uplus y=(x \cup y) \uplus(x \cap y))$ since, in general, $n+m=\max (n, m)+\min (n, m)$. Therefore, in particular, $n+0=\max (n, 0)$, so that we have $\forall x \forall y(x \cap y=\varnothing \leftrightarrow x \cup y=x \uplus y)$. Since $x \cap\{x\}=\varnothing$ by Axiom VI, $x \cup\{x\}=x \uplus\{x\}$. In general, however, $\cap x=\varnothing$ does not imply $\cup x=\uplus x$. For example, consider the mset $x=\left\{\{z\},\{y\},[y]_{2}\right\}$ where $y \neq z$. We have $\cap x=\varnothing$ but $\cup x=[z, y]_{1,2}$ and $\uplus x=[z, y]_{1,3} \neq \cup x$.

The identities $(x \cap y)^{*}=x^{*} \cap y^{*}$ and $(x \cup y)^{*}=(x \uplus y)^{*}=x^{*} \cup y^{*}$ are easily proved in MST. However, in general, $x^{*} \cup y^{*} \neq x^{*} \uplus y^{*}$. For further identities in the algebra of multisets (distributive laws, De Morgan laws, etc.) the interested reader is referred to [17], p. 636, and [15], pp. 215-216. The binary operation $\uplus$ is 'stronger' than both $U$ and $\cap$ in the sense that $\uplus$ distributes over 
both $U$ and $\cap$ but $U$ and $\cap$ do not, in general, distribute over $\uplus$. When restricted to hereditary sets in MST, the algebra of msets is identical to the algebra of sets in ZFC.

We now define the relative complement using the separation schema. Since PA $\vdash \forall m \forall k(k<m \rightarrow \exists ! n(m=k+n))$, we denote this unique $n$ by $m-k$ which is defined for all $m$ and $k$ such that $k<m$. For an mset $z$ let $y^{\prime} \subseteq z$ and let $\varphi(x, n)$ be the wff $\left(x \in^{n} z \wedge x \notin y^{\prime}\right) \vee \exists m \exists k\left(x \in \in^{m} z \wedge x \in^{k} y^{\prime} \wedge k<m \wedge\right.$ $n=m-k) . \varphi(x, n)$ is 'functional'. The unique mset $y$ in Axiom $\mathrm{IX}_{\varphi}$ is called the relative complement of $y^{\prime}$ in $z$. For any mset terms $u$ and $v$ such that $v \subseteq$ $u$, the relative complement of $v$ in $u$ is denoted by $u-v$. Clearly, for all such mset terms $u$ and $v, u-v \subseteq u$. We note that if the mset $y^{\prime}$ above contains all copies of $x$ in $z$ (if $\exists m\left(x \in^{m} y^{\prime} \wedge x \in^{m} z\right.$ ) holds), then neither disjunct of $\varphi(x, n)$ is satisfied and, therefore, $x \notin z-y^{\prime}$. One can show that $\forall y(y-\varnothing=$ $y \wedge y-y=\varnothing), \forall y \forall z \forall n\left(z \in^{n} y-y^{*} \leftrightarrow z \in^{n+1} y\right)$, and $\forall x \forall y\left(\forall z \forall n\left(z \in^{n} x \rightarrow\right.\right.$ $\left.\left.z \in^{n} y\right) \leftrightarrow \forall z \forall n\left(z \in^{n} y-x \rightarrow z \in^{n} y\right)\right)$ hold, the last of which states that " $x$ is whole in $y$ iff $y-x$ is whole in $y "$.

Unlike ZFC where elements occur at most once, in MST an element may occur in an msubset and in its relative complement. If this is the case, the nature of indistinguishability dictates that we cannot distinguish between copies in the msubset and copies in its relative complement. We can determine how many copies belong to each, but we cannot identify which copies are which.

In MST, therefore, there are many msets $x$ and $y$ for which $x \subseteq y \wedge(x \cap$ $(y-x) \neq \varnothing)$ holds. Hickman characterizes this situation as "unintuitive," "unattractive," "disturbing" and "inconsiderate" ([15], pp. 212, 214, 216). His distress is difficult to understand. The fact that a subset and its relative complement are disjoint in ZFC is simply a special case of the more general situation in MST. Therefore, $\langle\mathbb{P}(y), \cup, \cap,-, \varnothing, y\rangle$ is not a Boolean algebra, since for $x \in \mathbb{P}(y), x \cap(y-x) \neq \varnothing$ and $x \cup(y-x) \neq y$. However, $x \uplus(y-x)=$ $y$ always holds.

If the complementation operator is restricted to sets, then all is well; that is, $(\operatorname{Set}(y) \wedge x \subseteq y) \rightarrow(\operatorname{Set}(x) \wedge \operatorname{Set}(y-x) \wedge x \cap(y-x)=\varnothing \wedge x \cup(y-x)=$ $y$ ). If $x$ is whole in $y$, then $x \cap(y-x)=\varnothing$. If $x \cap(y-x)=\varnothing$ then $x \cup$ $(y-x)=x \uplus(y-x)$. Therefore, if $x$ is whole in $y$, both $x \cap(y-x)=\varnothing$ and $x \cup(y-x)=y$ hold. The complementation operator behaves classically when restricted to sets and whole msubsets. Whole msubsets are 'set-like' in the sense that elements do not spill over into the relative complement.

The replacement schema of MST states that for every wff $\varphi(x, y)$ of $L$ with free variables including $x$ and $y$ but excluding $y^{\prime}$ and $z^{\prime}$, the universal closure of

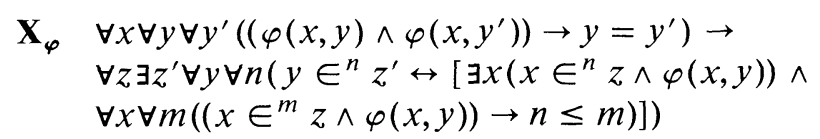

is an axiom of MST.

In words, Axiom $\mathrm{X}_{\varphi}$ states that if the wff $\varphi(x, y)$ is 'functional', then for any mset $z$ there is a unique (by Axiom II) mset $z^{\prime}$ that is the 'image' of $z$ under $\varphi$. If there is more than one $x$ in $z$ that is 'mapped' to some $y$ by $\varphi$, then the multiplicity $n$ of $y$ in $z^{\prime}$ is the least multiplicity of all $x$ in $z$ such that $\varphi(x, y)$.

The 'least multiplicity' condition is necessary because without it, if we were 
to replace two distinct elements $x$ and $x^{\prime}$ of $z$ with different multiplicities in $z$ by the same mset $y$ (using, for example, the wff $\varphi(x, y)$ as $x=x \wedge y=\varnothing$ ), then the mset $y$ would have two different multiplicities in $z^{\prime}$, contradicting Axiom I. We could have required that $\varphi(x, y)$ be ' $1-1$ ' $\left(\forall x \forall x^{\prime} \forall y\left(\left(\varphi(x, y) \wedge \varphi\left(x^{\prime}, y\right) \rightarrow\right.\right.\right.$ $\left.x=x^{\prime}\right)$ ) but this is too strong a condition on $\varphi(x, y)$.

Ignoring the effects of the 'least multiplicity' condition for a moment, axioms of the form $\mathrm{X}_{\varphi}$ represent exact replacement: elements in the 'range' occur with the exact multiplicity as do their 'pre-images' in the 'domain.' If downward replacement is required (if $n$ copies of $x$ in $z$ are to be replaced by fewer than $n$ copies of $y$ in $z^{\prime}$ ), the separation schema must be used first to 'scale down' the 'domain' to the required multiplicities, and then exact replacement will result in the desired 'range' mset.

The form of the replacement axioms $\mathrm{X}_{\varphi}$ does not permit us to deduce the separation schema from the replacement schema in the usual way. The multiplicity of some element in the 'range' mset $z$ ' must be the multiplicity of some 'associate' mset in the 'domain' mset $z$. Thus, if the 'domain' is to be a parent mset and the 'range' a possible msubset, one can never generate nonempty proper msubsets.

Since elements of $z$ are replaced by at most the same number of elements in $z^{\prime}$, the mset $z^{\prime}$ is 'limited in size' by the given mset $z$. We note also that in axioms of the form $\mathrm{X}_{\varphi}$, if $\operatorname{Set}(z)$ then $\operatorname{Set}\left(z^{\prime}\right)$. The converse is not true, since 'least multiplicity' may have been invoked.

If we attempt to construct an mset containing an element with infinite multiplicity by replacing every element of an infinite mset $z$ (Axiom XI) by $\varnothing$, for example, we would fail. Every element $x$ in $z$ has finite multiplicity in $z$ and there is a least such multiplicity $n$. The 'least multiplicity' condition gives $z^{\prime}=[\varnothing]_{n}$.

We will shortly define the cardinality of a finite mset to be the sum of the cardinalities of its simple whole parts. To justify this definition we now prove that every mset is the union of its simple disjoint whole msubsets:

Theorem $\forall x\left(x=\cup\left\{x_{y} \mid y \in x^{*}\right\}\right)$.

Proof: Let $x$ be some mset. Then every $x_{y} \subseteq x$ exists and $\operatorname{Set}\left(x^{*}\right)$ by the separation schema. We 'replace' each $y \in x^{*}$ by the mset $x_{y}$. The result is the set $z=\left\{x_{y} \mid y \in x^{*}\right\}$. Then $\cup z=\cup\left\{x_{y} \mid y \in x^{*}\right\}$ is an mset by Axiom VII. Since $\forall x \forall y \forall n\left(y \in \in^{n} x \leftrightarrow y \in^{n} x_{y}\right), x=\bigcup\left\{x_{y} \mid y \in x^{*}\right\}$ by Axiom II.

We now prove the existence of cross products in MST:

Theorem $\forall z \forall z^{\prime \prime} \exists z^{\prime \prime \prime} \forall x \forall y \forall l\left(\langle x, y\rangle \in \in^{l} z^{\prime \prime \prime} \leftrightarrow \exists n \exists m\left(x \in \in^{n} z \wedge y \in^{m} z^{\prime \prime} \wedge l=\right.\right.$ $n \cdot m))$. In words, for any msets $z$ and $z^{\prime \prime}$ there is an mset $z^{\prime \prime \prime}$ whose elements are ordered pairs $\langle x, y\rangle$. The multiplicity of each pair $\langle x, y\rangle$ in $z^{\prime \prime \prime}$ is the product of the multiplicity of $x$ in $z$ and the multiplicity of $y$ in $z^{\prime \prime}$.

Proof: Let $z$ and $z^{\prime \prime}$ be arbitrarily given msets, and $x$ an arbitrary but fixed element of $z$. Apply replacement to the mset $z^{\prime \prime}$ as follows: replace each $y$ in $z^{\prime \prime}$ by $\langle x, y\rangle$. Clearly this is a 1-1 replacement. If we call the result of this replacement $\{x\} \times z^{\prime \prime}$, we have $y \in \in^{m} z^{\prime \prime} \leftrightarrow\langle x, y\rangle \in^{m}\{x\} \times z^{\prime \prime}$. We now apply replacement to the mset $z$ : replace each $x$ in $z$ by the mset $\{x\} \times z^{\prime \prime}$ and call the result of this replacement $z^{\prime}$. Then we have $x \in \in^{n} z \leftrightarrow\{x\} \times z^{\prime \prime} \in \in^{n} z^{\prime}$ since this is also 
a $1-1$ replacement. Put $z^{\prime \prime \prime}=\uplus z^{\prime}$. It is then easy to show that $\langle x, y\rangle \in^{l} z^{\prime \prime \prime} \leftrightarrow$ $x \in \in^{n} z \wedge y \in \in^{m} z^{\prime \prime} \wedge l=n \cdot m$.

The mset $z^{\prime \prime \prime}$ above is unique by Axiom II and is called the cross product of $z$ and $z^{\prime \prime}$. For mset terms $u$ and $v$, the cross product of $u$ and $v$ is denoted by $u \times v$. In general, $x \times y \neq y \times x$ but $x \times y \approx y \times x$.

\section{Theorem Axiom IV(ii) is provable from the other axioms of MST.}

Proof: The set $\varnothing$ exists by Axiom III. The set $\mathbb{P}(\mathbb{P}(\varnothing))$ exists by Axiom V. Given msets $x$ and $y$ such that $x \neq y$ and numbers $n$ and $m$, the msets $[x]_{n}$ and $[y]_{m}$ exist by Axiom IV(i). Using the wff $\varphi\left(x^{\prime}, y^{\prime}\right)$ as $\left(x^{\prime}=\varnothing \rightarrow y^{\prime}=[x]_{n}\right) \wedge$ $\left(x^{\prime}=\{\varnothing\} \rightarrow y^{\prime}=[y]_{m}\right)$ the replacement Axiom $\mathrm{X}_{\varphi}$ applied to $\mathbb{P}(\mathbb{P}(\varnothing))$ gives the mset $z^{\prime}$, where $[x]_{n} \in{ }^{1} z^{\prime} \wedge[y]_{m} \in{ }^{1} z^{\prime} \wedge \forall z\left(z \in z^{\prime} \leftrightarrow z=[x]_{n} \vee z=[y]_{m}\right)$. The mset $\cup z^{\prime}$ is the required mset $z$ in Axiom IV(ii) which we defined to be $[x, y]_{n, m}$. Hence, $\forall x \forall y\left(x \neq y \rightarrow \forall n \forall m \exists z\left(x \in^{n} z \wedge y \in^{m} z \wedge \forall z^{\prime}\left(z^{\prime} \in z \leftrightarrow\right.\right.\right.$ $\left.\left.\left.\left(z^{\prime}=x \vee z^{\prime}=y\right)\right)\right)\right)$.

One could argue that the notion of 'exact replacement' would be better served if the replacement schema had the following effect: the multiplicity of $y$ in the range equals the sum of the multiplicities of all $x$ 's in the domain such that $\varphi(x, y)$ holds, if the sum is finite. Otherwise the maximum of the multiplicities of the preimages is used, if it exists. If the sum is not finite and the maximum does not exist, then the minimum of the multiplicities of the preimages is used. One could revise Axioms $\mathrm{X}_{\varphi}$ to give such 'additive replacement' axioms. However, using Axioms $\mathrm{X}_{\varphi}$, a theorem schema of 'additive replacement' is provable in MST (the proof is difficult and lengthy), [3], pp. 97-101.

The infinite mset axiom of MST is

XI $\exists y(\varnothing \in y \wedge \forall x(x \in y \rightarrow x \cup\{x\} \in y))$.

One can show (exactly as in ZFC, see [10], p. 47), that if $\mathbb{N}$ is the smallest (with respect to $\subseteq$ ) mset $y$ that satisfies Axiom XI, then $H \operatorname{Set}(\mathbb{N}), \operatorname{Lim}(\mathbb{N})$, and $\mathbb{N}=$ $\{\varnothing,\{\varnothing\},\{\varnothing,\{\varnothing\}\}, \ldots\}=\{x \mid \operatorname{VNN}(x)\}$.

Using the separation schema with $\varphi(x, n)$ as $x \neq \varnothing \wedge n=1$ we obtain the set $\overline{\mathbb{N}}=\{\{\varnothing\},\{\varnothing,\{\varnothing\}\}, \ldots\} \subseteq \mathbb{N}$. Using the wff $x=\hat{n} \wedge y=[z]_{n}$ as $\varphi(x, y)$ in the replacement schema, we obtain the infinite set $\left\{\{z\},[z]_{2},[z]_{3}, \ldots\right\}$ which provided useful examples when we discussed union, intersection, and additive union. Call this infinite set $x$. Then $\cup x=\uplus x=\cap x=\{z\}$ since the only multiplicity of $z$ as an element of elements of $x$ that exists is its minimum multiplicity. We have proved $\forall z \exists y\left(\operatorname{Set}(y) \wedge \forall x\left(x \in y \leftrightarrow \exists n x=[z]_{n}\right)\right)$. Therefore, for a given mset $z$, the collection of all simple msets of the form $[z]_{n}$ (that exist by Axiom IV(i)), is itself a set in MST.

We now construct the mset $T C(x)$. Given the mset $x$ and the infinite set $\mathbb{N}$ (defined above), we replace each $\hat{n}$ in $\mathbb{N}$ by the mset $\cup^{\hat{n}} x$ where $\cup^{\hat{0}} x=x, \cup^{\hat{1}} x=$ $\cup x, \cup^{\widehat{n+1}} x=\cup \cup^{\hat{n}} x$. Since $\mathbb{N}$ is analogous to the classical set $\omega$ in ZFC, we have the standard recursion theorem on $\mathbb{N}$. The replacement wff $\varphi(\hat{n}, y, x)$ is $y=\cup^{\hat{n}} x$ where $x$ is a parameter. We note that our recursive definition is such that $\forall n \forall z\left(z \in \cup^{n+1} x \leftrightarrow \exists z^{\prime}\left(z^{\prime} \in \cup^{\hat{n}} x \wedge z \in z^{\prime}\right)\right.$. We call the result of this replacement the mset $\mathbb{N}^{\prime}$. The elements of $\mathbb{N}^{\prime}$ are $x, \cup x, \cup \cup x, \ldots$. Using the wff $y=x^{*}$, we replace each element of $\mathbb{N}^{\prime}$ by its root set to obtain the mset $\mathbb{N}^{\prime \prime}$. The elements of $\mathbb{N}^{\prime \prime}$ are $x^{*},(\cup x)^{*},(\cup \cup x)^{*}, \ldots$ Define $T C(x)=\cup \mathbb{N}^{\prime \prime}$. Infor- 
mally, therefore, the mset $T C(x)$ is $x^{*} \cup(\cup x)^{*} \cup(\cup \cup x)^{*} \cup$.. which contains the elements of $x$, the elements of elements of $x$, . etc. Every element of $T C(x)$ occurs at most once in $T C(x)$ so that $\operatorname{Set}(T C(x))$. Clearly $x^{*} \subseteq T C(x)$. If $y \in z$ and $z \in T C(x)$, then $\exists n\left(z \in\left(\cup^{n} x\right)^{*} \wedge y \in\left(\cup^{n+1} x\right)^{*}\right)$ and, therefore, $y \in T C(x)$. Hence $\operatorname{Trans}(T C(x)) . T C(x)$ is the smallest transitive set with $x^{*}$ as an msubset, and we have $\forall x T C(x)=T C\left(x^{*}\right)$.

Theorem $\quad \forall x((\operatorname{Set}(x) \wedge \operatorname{Trans}(x)) \leftrightarrow T C(x)=x)$.

Proof: The $\leftarrow$ direction is obvious. For the $\rightarrow$ direction, let $x$ be such that Set $(x) \wedge \operatorname{Trans}(x)$ holds. Since $x=x^{*}$ and $\forall x\left(x^{*} \subseteq T C(x)\right), x \subseteq T C(x)$. To show that $T C(x) \subseteq x$, let $z \in T C(x)$ and assume $z \notin x$. Then $\{z \in T C(x) \mid z \notin$ $x\} \neq \varnothing$. To each such $z, \exists n_{z} \geq 1$ such that $z \in\left(\cup^{n_{z}} x\right)^{*}$, but $\forall n<n_{z} z \notin$ $\left(\cup^{n} x\right)^{*}$. (Each $n_{z} \geq 1$, since otherwise $z \in x^{*}$. Recall also that $T C(x)=x^{*} \cup$ $\left.(\cup x)^{*} \cup\left(\cup^{2} x\right)^{*} \cup \ldots\right)$ We pick a particular $\bar{z} \in T C(x)$ such that $\bar{z} \notin x$, but $n_{\bar{z}}$ is least among the $n_{z}$ 's. Therefore $\bar{z} \in\left(\cup^{n_{\bar{z}}} x\right)^{*} \wedge n_{\bar{z}} \geq 1$. Therefore, $\exists y(y \in$ $\left.\left(\cup^{n_{\bar{z}}-1} x\right)^{*} \wedge \bar{z} \in y\right)$ holds.

If $n_{\bar{z}}=1$ then $y \in x^{*}$. Hence $\bar{z} \in y \wedge y \in x \rightarrow \bar{z} \in x$ since $\operatorname{Trans}(x)$. But $\bar{z} \notin x$. Therefore, $n_{\bar{z}}>1$.

If $y \notin x$, then $y \in T C(x) \wedge y \notin x \wedge y \in\left(\cup^{n_{\bar{z}}-1} x\right)^{*}$, so that $n_{y}<n_{\bar{z}}$ which contradicts that $n_{\bar{z}}$ is a least $n_{z}$.

Hence, $y \in x$ and $\bar{z} \in y \wedge y \in x \rightarrow \bar{z} \in x$ since Trans $(x)$. But $\bar{z} \notin x$. Therefore, $\{z \in T C(x) \mid z \notin x\}=\varnothing$ and $T C(x) \subseteq x$. Hence $T C(x)=x$ as required. In particular, therefore, since $\forall n(\operatorname{Set}(\hat{n}) \wedge \operatorname{Trans}(\hat{n}))$ we have $\forall n \operatorname{TC}(\hat{n})=\hat{n}$.

The choice mset axiom of MST is

XII $\forall y[[y \neq \varnothing \wedge \forall x(x \in y \rightarrow x \neq \varnothing) \wedge$

$$
\begin{aligned}
& \forall x \forall z((x \in y \wedge z \in y \wedge x \neq z) \rightarrow x \cap z=\varnothing)] \rightarrow \\
& \exists y^{\prime}\left(\forall x \forall n \left(x \in n \in y \rightarrow \exists x ^ { \prime } \left(x^{\prime} \in{ }^{n} y^{\prime} \wedge x^{\prime} \in x \wedge\right.\right.\right. \\
& \left.\forall x^{\prime \prime}\left(\left(x^{\prime \prime} \in x \wedge x^{\prime \prime} \in y^{\prime}\right) \rightarrow x^{\prime \prime}=x^{\prime}\right)\right) \wedge \\
& \left.\left.\forall x^{\prime} \forall n\left(x^{\prime} \in \in^{n} y^{\prime} \rightarrow \exists x\left(x \in{ }^{n} y \wedge x^{\prime} \in x\right)\right)\right)\right] .
\end{aligned}
$$

Any mset $y^{\prime}$ that satisfies Axiom XII is called a choice mset for $y$. The elements $x^{\prime}$ in $y^{\prime}$ occur with the same multiplicity as do their corresponding elements $x$ in the mset $y$. The multiplicities of the 'chosen' elements $x^{\prime}$ in $x$ do not matter. It is obvious that a choice mset $y^{\prime}$ for an mset $y$ is not unique unless, of course, every element $x$ of $y$ is a simple mset (when only one 'choice' for each $x^{\prime} \in x$ is possible). If we add the condition Set (y) to Axiom XII, then $y^{\prime}$ is called a choice set for $y$ since $\operatorname{Set}\left(y^{\prime}\right)$. If we add the condition $\operatorname{HSet}(y)$ to Axiom XII, then HSet $\left(y^{\prime}\right)$ and the resulting wff is equivalent to the classical axiom of choice (AC) of ZFC.

\section{Theorem XII is equivalent to}

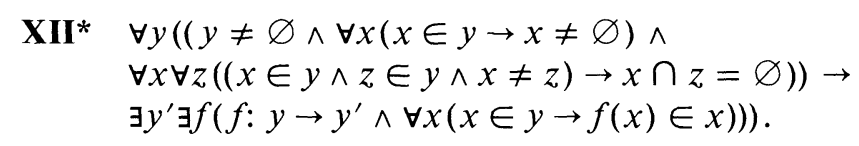

Proof: XII implies XII* since for the given mset $y$ there exists a choice mset $y^{\prime}$. For each $x \in y$, there is a unique mset $x^{\prime}$ such that $\left\{x^{\prime}\right\}=x \cap\left(y^{\prime}\right)^{*}$. We define the function $f: y^{*} \rightarrow\left(y^{\prime}\right)^{*}$ by the rule: for each $x \in y^{*}$, let $f(x) \in x \cap\left(y^{\prime}\right)^{*}$. Then $f: y \rightarrow y^{\prime}$ and $\forall x(x \in y \rightarrow f(x) \in x)$. XII* implies XII since for the given mset $y$ there is a choice function $f$ on $y$. Let $\operatorname{ran} f=\left\{z^{\prime} \mid\left\langle z, z^{\prime}\right\rangle \in f\right\}$. If we replace 
each element $f(x)$ in $\operatorname{ran}^{*}$ by $[f(x)]_{n}$ iff $x \in^{n} y$ and call the result $y^{\prime \prime}$, then the mset $U y^{\prime \prime}$ is the required choice mset $y^{\prime}$ in Axiom XII.

Theorem $X I I$ is equivalent to

XII** $\forall y((y \neq \varnothing \wedge \forall x(x \in y \rightarrow x \neq \varnothing)) \rightarrow$

$\left.\exists y^{\prime} \exists f\left(f: y \rightarrow y^{\prime} \wedge \forall x(x \in y \rightarrow f(x) \in x)\right)\right)$.

Proof: Since $\mathrm{XII}^{* *}$ obviously implies XII* which implies XII, XII** implies XII. To show that XII implies XII** we mimic the classical proof in [10], p. 56. Let $y \neq \varnothing$ be an mset that does not contain $\varnothing$ as an element. We replace each $x$ in $y$ by the mset $\{x\} \times x$ and call the result $y^{\prime \prime}$. Since this is an exact 1-1 replacement, $\forall x \forall n\left(x \in \in^{n} y \leftrightarrow\{x\} \times x \in^{n} y^{\prime \prime}\right)$. Since $y \neq \varnothing, y^{\prime \prime} \neq \varnothing$. Since $\forall x(x \in y \rightarrow x \neq \varnothing)$ we have $\forall x\left(\{x\} \times x \in y^{\prime \prime} \rightarrow\{x\} \times x \neq \varnothing\right)$. If $\{x\} \times x \neq$ $\{z\} \times z$ then $x \neq z$. If $(\{x\} \times x) \cap(\{z\} \times z) \neq \varnothing$, then there is an element $\left\langle z^{\prime}, z^{\prime \prime}\right\rangle$ such that $\left\langle z^{\prime}, z^{\prime \prime}\right\rangle \in\{x\} \times x$ and $\left\langle z^{\prime}, z^{\prime \prime}\right\rangle \in\{z\} \times z$. If this is the case, then $z^{\prime} \in\{x\}$ and $z^{\prime} \in\{z\}$ and, therefore, $z^{\prime}=x=z$ which contradicts our choice of $x$ and $z$. Hence, distinct elements of $y^{\prime \prime}$ are disjoint, and the mset $y^{\prime \prime}$ satisfies the conditions in the antecedent of Axiom XII. By Axiom XII, $y^{\prime \prime}$ has a choice mset $\bar{f}$ such that:

$$
\forall x \forall n\left(x \in^{n} y \leftrightarrow\{x\} \times x \in^{n} y^{\prime \prime} \leftrightarrow\left\langle x, x^{\prime}\right\rangle \in^{n} \bar{f}\right),
$$

where $\left\langle x, x^{\prime}\right\rangle \in\{x\} \times x$. We have, therefore, $\forall x\left(x \in y \rightarrow\left(\left\langle x, x^{\prime}\right\rangle \in \bar{f} \wedge x^{\prime} \in x\right)\right)$. Let $f=(\bar{f})^{*}$ and let $y^{\prime}=\left\{x^{\prime} \mid\left\langle x, x^{\prime}\right\rangle \in f\right\}$. Clearly, $f$ is a function from $y^{*}$ to $y^{\prime}$ so that $f: y \rightarrow y^{\prime}$. We have proven, therefore, that

$$
\exists y^{\prime} \exists f\left(f: y \rightarrow y^{\prime} \wedge \forall x(x \in y \rightarrow f(x) \in x)\right) .
$$

Therefore, XII implies XII**.

We define a translation ' from wffs $\varphi$ of ZFC to wffs $\varphi^{\prime}$ of $L$ as follows:

$$
\begin{aligned}
& (x=y)^{\prime} \text { is } H \operatorname{Set}(x) \wedge H \operatorname{Set}(y) \wedge x=y \\
& (x \in y)^{\prime} \text { is } H \operatorname{Set}(x) \wedge H \operatorname{Set}(y) \wedge x \in^{1} y \\
& (\sim \psi)^{\prime} \text { is } \sim \psi^{\prime},(\psi \vee \theta)^{\prime} \text { is } \psi^{\prime} \vee \theta^{\prime} \\
& (\psi \rightarrow \theta)^{\prime} \text { is } \psi^{\prime} \rightarrow \theta^{\prime},(\psi \leftrightarrow \theta)^{\prime} \text { is } \psi^{\prime} \leftrightarrow \theta^{\prime} \\
& (\exists x \psi)^{\prime} \text { is } \exists x\left(\operatorname{HSet}(x) \wedge \psi^{\prime}\right) \\
& (\forall x \psi)^{\prime} \text { is } \forall x\left(\operatorname{HSet}(x) \rightarrow \psi^{\prime}\right) .
\end{aligned}
$$

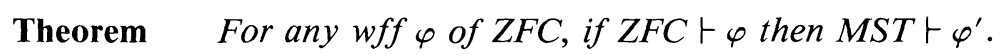

Proof: If $\varphi$ is an axiom of $\mathrm{ZFC}$, then $\varphi^{\prime}$ is an obvious special case of an axiom of MST and is, therefore, a theorem of MST. The ' translation preserves rules of inference, so that the ' translation of a proof of $\varphi$ in ZFC is a proof of $\varphi^{\prime}$ in MST.

If $\mathrm{ZFC}^{\prime}=\left\{\varphi^{\prime} \mid \varphi \in \mathrm{ZFC}\right\}$, then the theory MST contains a copy ZFC' of ZFC, that is, $\mathrm{ZFC}^{\prime} \subset \mathrm{MST}$.

If we had translated the atomic formula $x \in y$ of $\mathrm{ZFC}$ as $\operatorname{Set}(x) \wedge \operatorname{Set}(y) \wedge$ $x \in{ }^{1} y$ in MST, then the translations of axioms of ZFC would not, in general, be theorems of MST. For example, the translation of the classical axiom of extensionality would be $\forall x \forall y\left(\forall z\left(\left(\operatorname{Set}(z) \wedge \operatorname{Set}(x) \wedge z \in^{1} x\right) \leftrightarrow(\operatorname{Set}(z) \wedge \operatorname{Set}(y) \wedge\right.\right.$ $\left.\left.\left.z \in^{1} y\right)\right) \rightarrow x=y\right)$. This is clearly false in MST. For example, consider the sets $\{x, y\}$ and $\{x\}$ where $x \neq y, \operatorname{Set}(x)$ and $\sim \operatorname{Set}(y)$. They satisfy the antecedent but they are certainly not equal.

With $\mathrm{ZFC}^{\prime}$ at hand, we proceed (exactly as we would in $\mathrm{ZFC}$ ) to construct 
the cumulative hierarchy $V=\bigcup_{\alpha \in O n} V_{\alpha}$, the class $O n$ of ordinals, the class Card of cardinal numbers, etc. The axiom of foundation of ZFC (a theorem of MST) proves that $V=\bigcup_{\alpha \in O n} V_{\alpha}=\{x \mid H \operatorname{Set}(x)\}$. (See, for example, [10], p. 94). Therefore, the hsets of MST are exactly the elements of $V$.

We can also construct an $M_{\alpha}$ hierarchy for multisets. If we define, for each $\alpha \in$ On, $M_{\alpha}=\left\{x \mid x^{*} \in V_{\alpha}\right\}$ we would obtain only msets whose elements are hsets. Under this definition, for example, we would 'miss' all msets with root set $\left\{[\varnothing]_{2}\right\}$. In order to 'catch' all msets, we define $M$ as follows: $M_{0}=\varnothing, M_{\alpha+1}=\left\{x \mid x^{*} \in \mathbb{P}\left(M_{\alpha}\right)\right\} ; M_{\lambda}=\bigcup_{\alpha<\lambda} M_{\alpha}$ if $\lambda$ is a limit ordinal; and $M=\bigcup_{\alpha \in O n} M_{\alpha}$. To prove that we have indeed 'caught' all msets of MST, suppose to the contrary that $\exists x x \notin M$ holds. The class term $\{y \mid y \in$ $T C(\{x\}) \wedge y \notin M\} \subseteq T C(\{x\})$ is an mset $z$. Since $x \in T C(\{x\}) \wedge x \notin M$ we have $z \neq \varnothing$. By Axiom VI, there exists an $\in$-minimal element $x^{\prime}$ in $z\left(x^{\prime} \in z \wedge x^{\prime} \cap\right.$ $z=\varnothing)$. Hence every element of $x^{\prime}$ is in $M$. We replace each element of $\left(x^{\prime}\right)^{*}$ by its ordinal rank in $M$. The union of the resulting mset is the ordinal $\beta$. Every element of $\left(x^{\prime}\right)^{*}$ is an element of $M_{\beta}$, so $\left(x^{\prime}\right)^{*} \subseteq M_{\beta}\left(\left(x^{\prime}\right)^{*} \in \mathbb{P}\left(M_{\beta}\right)\right)$. If this is the case, $x^{\prime} \in M_{\beta+1}$ which contradicts $x^{\prime} \notin M$. Therefore, $\forall x x \in M$ holds. The multiset universe $\{x \mid x=x\}$ of MST is exactly the class $M$.

In order to define the cardinality of msets we require an operation $H$ such that $\forall x H \operatorname{Set}(H(x))$ and $\forall x \forall y(x \neq y \rightarrow H(x) \neq H(y))$. The operation $H$ when applied to distinct msets should give distinct hereditary sets. Distinct msets either contain distinct elements, or contain an element in common with distinct multiplicities. This elementary fact suggests the following definition: for all mset terms $u$ and $v$, define

$$
H(v)=\left\{\langle H(u), \hat{m}\rangle \mid u \in^{n} v \wedge 1 \leq m \leq n\right\} .
$$

For example,

$$
\begin{aligned}
& H(\varnothing)=\varnothing, \\
& H(\{\varnothing\})=\{\langle H(\varnothing), \hat{1}\rangle\}, \\
& H\left([\varnothing]_{2}\right)=\{\langle H(\varnothing), \hat{1}\rangle,\langle H(\varnothing), \hat{2}\rangle\}, \\
& H(\{\{\varnothing\}\})=\{\langle H(\{\varnothing\}), \hat{1}\rangle\}, \\
& H\left(\left[[\varnothing]_{2}\right]_{2}\right)=\left\{\left\langle H\left([\varnothing]_{2}\right), \hat{1}\right\rangle,\left\langle H\left([\varnothing]_{2}\right), \hat{2}\right\rangle\right\}, \\
& H\left([\varnothing,\{\varnothing\}]_{2,3}\right)=\{\langle H(\varnothing), \hat{1}\rangle,\langle H(\varnothing), \hat{2}\rangle,\langle H(\{\varnothing\}), \hat{1}\rangle, \\
& \\
& \qquad H(\{\varnothing\}), \hat{2}\rangle,\langle H(\{\varnothing\}), \hat{3}\rangle\} .
\end{aligned}
$$

It is straightforward to show that $H$ is well-defined and 1-1.

Theorem $\forall y \operatorname{HSet}(H(y))$.

Proof: $H(y)=\left\{\langle H(x), \hat{m}\rangle \mid x \in^{n} y \wedge 1 \leq m \leq n\right\}$.

We prove $\forall y H \operatorname{Set}(H(y))$ by induction on the rank of $y$ in $M$. If the rank of $y$ is 1 , then $y=\varnothing, H(\varnothing)=\varnothing$, and $H \operatorname{Set}(\varnothing)$. We assume $H \operatorname{Set}(H(x))$ for all $x \in M$ with $r(x) \leq \beta$. Let $r(y)=\beta+1$. To show that $H \operatorname{Set}(H(y))$, we note that $H(y)$ is a set of ordered pair sets by definition. Each first component is an hset by the induction hypothesis. Each second component is an hset since $\forall n$ $\operatorname{HSet}(\hat{n})$. Therefore, the only way in which $H(y)$ could fail to be hereditary is if some $\langle H(x), \hat{m}\rangle$ in $H(y)$ is such that $H(x)=\hat{m}$ (since then $\langle H(x), \hat{m}\rangle=$ $\left.\left\{\{H(x)\},[H(x)]_{2}\right\}\right)$. However, this can never happen, since all $H(x)$ are sets of 
ordered pairs, but $\hat{m}$ for $m \geq 1$ is never a set of ordered pairs. Therefore, $H(y)$ is an hereditary set, and $H \operatorname{Set}(H(y))$ for every mset $y$ in $M$.

We first define the cardinality of hsets, and then the cardinality of msets in general. For any mset $u$ such that $H \operatorname{Set}(u)$, we define the cardinality of $u$, written $C(u)$, to be the cardinal $\kappa \in C$ ard assigned to $u$ in our copy ZFC' of ZFC. There are several ways in which this assignment can be made depending upon the presence or absence of AC ([21], pp. 83-84). Since we assume AC throughout, $C(u)$ is the least ordinal equinumerous to $u$. For any mset $u$, we define the cardinality of $u$, written $C(u)$, by $C(u)=C(H(u))$.

An mset $u$ is infinite if $C(u) \geq \aleph_{0}$ (where $\geq$ is the ordering of the cardinals in ZFC'). An mset $u$ is finite if $C(u)<\aleph_{0}$, or equivalently, if $u=\varnothing \vee \exists n$ $C(u)=\hat{n}$ holds. If $\operatorname{Set}(u)$, then $u \approx H(u)$. If $\sim \operatorname{Set}(u)$ and $u$ is finite, then repeated elements in $u$ are counted as distinct elements in $H(u)$. If $u$ is infinite, then $C(u)=C\left(u^{*}\right)$ since each element of $u$ contributes at most a finite number (its multiplicity) to $C(u)$.

In general, for all msets $x, C(x) \geq C\left(x^{*}\right)$. If $C(x)=C\left(x^{*}\right)$, then either $x$ is infinite or $\operatorname{Set}(x)$ holds. If $C(x)>C\left(x^{*}\right)$ then $x$ is finite and $\sim \operatorname{Set}(x)$ holds. One can show that $\forall x(\forall y \forall z((y \in x \wedge z \in x) \rightarrow y=z) \rightarrow(C(x)=$ $\hat{0} \vee \exists n C(x)=\hat{n})$, that is, every simple mset is finite. Other theorems include: $\forall x \forall y(x \leq y \rightarrow C(x) \leq C(y)), \forall x \forall y(x \subseteq y \rightarrow C(x) \leq C(y)), \forall x \forall y((x \leq y \wedge$ $y \leq x) \rightarrow C(x)=C(y))$ and $\forall x \forall y(x \approx y \rightarrow C(x)=C(y))$. However, all the converses are false-consider the two msets $[x, y]_{2,1}$ and $\{x, y, z\}$.

The theory MST can be modified to allow urelements by methods similar to those used for ZFC. There are several ways in which this could be done (see, for example, [10], p. 25 , footnote 1 . One could also adapt the technique used in [2], pp. 7-11.)

It is important to note that our two-sorted approach to the theory MST provides a general strategy for the formalization of a whole family of nonclassical set theories. Instead of the axioms of Peano Arithmetic, one could use axioms (stated in numeric variable symbols) for a variety of algebraic structures.

3 A model of $M S T$ To establish the relative consistency of MST, we construct a model of MST in ZFC by interpreting multisets as positive integer-valued functions. We assume ZFC throughout. Recall that the language $L$ of MST is $\{e, \hat{,}, \mathrm{S},+, \cdot, 0\}$. We construct an $L$-structure $\mathbb{F}$ with two domains: the set $\omega$ of finite ordinals and a hierarchical class $F$ of functions. The numeric variable symbols of $L$ are to range over the set $\omega$, and the mset variable symbols over the class $F$. Let $\bar{\omega}$ be the set $\omega$ less $\varnothing$. We define the class $F$ of $\bar{\omega}$-valued functions as follows: $F_{0}=\varnothing ; F_{\alpha+1}=\left\{x: \operatorname{dom} x \rightarrow \bar{\omega} \mid \operatorname{dom} x \subseteq F_{\alpha}\right\} ; F_{\lambda}=\bigcup_{\alpha<\lambda} F_{\alpha}$ if $\operatorname{Lim}(\lambda)$ and $F=\bigcup_{\alpha \in O n} F_{\alpha}$. In other words, the range (range $\left.(x)\right)$ of each function $x$ in $F$ is a subset of $\bar{\omega}$. The domain (dom $x)$ of each function $x$ in $F$ is a set of functions in $F$ of lesser rank than $x$ (where the rank $r(x)$ of $x$ in $F$ is the least ordinal $\alpha \in O n$ such that $x \in F_{\alpha}$-this is different from the usual rank function in ZFC).

Let us compare the first few levels of the $M_{\alpha}$ and $F_{\alpha}$ hierarchies: $F_{0}=$ $M_{0}=\varnothing, F_{1}=M_{1}=\{\varnothing\}, F_{2}=\{\varnothing,\{\langle\varnothing, 1\rangle\},\{\langle\varnothing, 2\rangle\}, \ldots,\{\langle\varnothing, n\rangle\}, \ldots\}$ 
whereas $M_{2}=\left\{\varnothing,\{\varnothing\},[\varnothing]_{2}, \ldots,[\varnothing]_{n}, \ldots\right\} . F_{2}$ contains a countably infinite number of functions. $F_{3}=\left\{x: \operatorname{dom} x \rightarrow \bar{\omega} \mid \operatorname{dom} x \subseteq F_{2}\right\}$ contains uncountably many functions of $F$ and contains the first functions of $F$ with countably infinite domains (for example, $x: F_{2} \rightarrow\{1\}$ ). Consider the function $x: F_{2} \rightarrow \bar{\omega}$ defined by $x(\varnothing)=1$ and $\forall n>0 x(\{\langle\varnothing, n\rangle\})=n$. This function $x$ in $F_{3}$ is the interpretation in $\mathbb{F}$ of the mset $\left[\varnothing,\{\varnothing\},[\varnothing]_{2},[\varnothing]_{3}, \ldots\right]_{1,1,2,3 \ldots}$ of MST (which is an element of $\left.M_{3}=\left\{x \mid x^{*} \in V_{3} \cup \mathbb{P}\left(M_{2}\right)\right\}\right)$. The function $x:\{\langle\langle\varnothing, 13\rangle\}\} \rightarrow$ $\{1066\}$ is in $F_{3}$ (since dom $x \subseteq F_{2}$ ) and is the interpretation in $\mathbb{F}$ of the mset [[ $\left.\varnothing]_{13}\right]_{1066}$ of MST (in $M_{3}$ ). The function $x:\{\varnothing,\{\langle\varnothing, 7\rangle\},\{\langle\varnothing, 1985\rangle\}\} \rightarrow\{1\}$ is in $F_{3}$ (since dom $x \subseteq F_{2}$ ) and is the interpretation of the set $\left\{\varnothing,[\varnothing]_{7},[\varnothing]_{1985}\right\}=$ $\left[\varnothing,[\varnothing]_{7},[\varnothing]_{1985}\right]_{1,1,1}$ of MST (in $M_{3}$ ). The sets of MST correspond to the subclass $\{x \in F \mid \operatorname{range}(x)=\{1\}\}$ of the class $F . F_{4}=\left\{x: \operatorname{dom} x \rightarrow \bar{\omega} \mid \operatorname{dom} x \subseteq F_{3}\right\}$ contains the first functions of $F$ with uncountably infinite domains (like $x: F_{3} \rightarrow$ $\{1\})$.

Equality in $F$ is equality of sets in ZFC: two functions $x$ and $y$ in $F$ are equal iff $x$ and $y$ are equal sets of ordered pairs. The two domains of $\mathbb{F}, \omega$ and $F$, are disjoint except for $\varnothing$. No element of $\bar{\omega}$ is a set of ordered pairs, but every element of $F$ is a set of ordered pairs.

Some useful facts about the structure of $F$ which we need in order to prove that $\mathbb{F}$ is a model of MST are: $r(x)$ is never a limit ordinal; if $y \in \operatorname{dom} x$ then $r(y)<r(x)$; if $r(x) \leq \alpha$, then $x \in F_{\alpha}$; if $\alpha \leq \beta$, then $F_{\alpha} \subseteq F_{\beta}$; for each $\alpha \in O n$, $F_{\alpha}=\{x \in F \mid r(x) \leq \alpha\}$; and for each $\alpha \in O n, F_{\alpha+1}=F_{\alpha} \cup\{x \in F \mid r(x)=$ $\alpha+1\}$.

Let $\varphi$ be a wff of L. The interpretation of $\varphi$ in $\mathbb{F}$, denoted by $\mathbb{F}(\varphi)$, is defined by induction on the logical complexity of $\varphi$ as follows: $\mathbb{F}(x=y)$ is $x \in F \wedge y \in F \wedge x=y, \mathbb{F}(n=m)$ is $n \in \omega \wedge m \in \omega \wedge n=m, \mathbb{F}(e(x, y, n))$ is $x \in F \wedge y \in F \wedge n \in \omega \wedge y(x)=n, \mathbb{F}(\sim \psi)$ is $\sim \mathbb{F}(\psi), \mathbb{F}(\psi \vee \theta)$ is $\mathbb{F}(\psi) \vee \mathbb{F}(\theta)$, $\mathbb{F}(\psi \rightarrow \theta)$ is $\mathbb{F}(\psi) \rightarrow \mathbb{F}(\theta), \mathbb{F}(\exists n \psi)$ is $\exists n(n \in \bar{\omega} \wedge \mathbb{F}(\psi)), \mathbb{F}(\forall n \psi)$ is $\forall n(n \in \bar{\omega} \rightarrow$ $\mathbb{F}(\psi)), \mathbb{F}(\exists x \psi)$ is $\exists x(x \in F \wedge \mathbb{F}(\psi))$, and $\mathbb{F}(\forall x \psi)$ is $\forall x(x \in F \rightarrow \mathbb{F}(\psi))$. Clearly, if $\varphi$ is a wff of $L$, then $\mathbb{F}(\varphi)$ is a wff of ZFC. By " $\varphi$ holds in $\mathbb{F}$ " we mean $\mathrm{ZFC} \vdash \mathbb{F}(\varphi)$.

In $\mathbb{F}(\varphi)$, numeric quantifiers are restricted to the set $\bar{\omega}$, and mset quantifiers to the class $F$. When writing $\mathbb{F}(\varphi)$ for some particular wff $\varphi$ of $L$, these restrictions on quantifiers are to be understood - they will not be written out explicitly. Elements of sets defined below are understood to be restricted to the class $F$ or the set $\bar{\omega}$, as appropriate.

Therefore, $\mathbb{F}\left(x \in \in^{n} y\right)$ is $y(x)=n$. Since $x \in y$ is $\exists n x \in{ }^{n} y, \mathbb{F}(x \in y)$ is $\exists n(n \in \bar{\omega} \wedge y(x)=n)$, or simply $x \in \operatorname{dom} y$. It is straightforward to show that $\mathbb{F}(\operatorname{Set}(x))$ is $x=\varnothing \vee \operatorname{range}(x)=\{1\}$ and that $\mathbb{F}(x \subseteq y)$ is dom $x \subseteq \operatorname{dom} y \wedge$ $\forall z(z \in \operatorname{dom} x \rightarrow x(z) \leq y(z))$. For any function $y$ in $F$, the function $z=$ $\{\langle x, 1\rangle \mid x \in \operatorname{dom} y\}$ in $F(r(z)=r(y)$ since dom $z=$ dom $y$ ) is the 'root set of $y^{\prime}$ in $\mathbb{F}$.

To interpret the unary function symbol ${ }^{\wedge}$ of $L$ we define a function also denoted by ${ }^{\hat{N}}$. We define $\hat{}{ }^{\prime}: \omega \rightarrow F$ by $\hat{n}=\{\langle\hat{m}, 1\rangle \mid m \in n\}$ for all $n \in \omega$. Equivalently, $\hat{0}=\varnothing$ and $n \hat{\cup}\{n\}=\hat{n} \cup\{\langle\hat{n}, 1\rangle\}$ for all $n \in \bar{\omega}$. So

$$
\begin{aligned}
& \hat{1}=\{\langle\varnothing, 1\rangle\} \text { and } \\
& \hat{2}=\{\langle\varnothing, 1\rangle,\langle\{\langle\varnothing, 1\rangle\}, 1\rangle\} .
\end{aligned}
$$


The function ${ }^{\wedge}: \omega \rightarrow F$ is a $1-1$ embedding of the set $\omega$ into the class $F$. One can show, for all $n \in \omega$, that $\hat{n} \in F_{n+1}$, so that the range of ${ }^{\wedge}$ is a subset of $F_{\omega}$. We will extend this function to $\hat{:} V \rightarrow \hat{V} \subset F$ shortly.

The other nonlogical symbols of $L(\mathrm{~S},+, \cdot, 0)$ are interpreted in $\mathbb{F}$ as the usual successor, addition, multiplication, and zero of the set $\omega$. In other words, for each $n \in \omega, \mathrm{S} n=n \cup\{n\}$, + is defined by Axioms N3 and N4, - is defined by Axioms N5 and N6, and $0=\varnothing$.

To prove that $\mathbb{F}$ is a model of MST, we prove that every axiom $\theta$ of MST holds in $\mathbb{F}$-that is, ZFC $\vdash \mathbb{F}(\theta)$. Our choice of $\omega$ and our definitions of ${ }^{\wedge}$, $\mathrm{S},+, \cdot$, and 0 are such that Axioms N1-N6, N8, and all induction Axioms $\mathrm{N}_{\varphi}$ hold in $\mathbb{F}$. With respect to Axiom N8, we note that singleton sets $\{\varnothing\}$ and $\{\hat{n}\}$ of MST become the functions $\{\langle\varnothing, 1\rangle\}$ and $\{\langle\hat{n}, 1\rangle\}$ in the model.

Axiom I holds in $\mathbb{F}$ simply because elements of $F$ are functions. Axiom II holds in $\mathbb{F}$ since two functions that agree at all arguments are equal. Axiom III holds in $\mathbb{F}$ since the empty function $\varnothing \in F_{1}$. Since $\{\langle x, n\rangle\}$ models $[x]_{n}$ and $\{\langle x, n\rangle,\langle y, m\rangle\}$ where $x \neq y$ models $[x, y]_{n, m}$, Axioms IV(i) and IV(ii) hold in $\mathbb{F}$. For Axiom $\mathrm{V}$ we note that for any function $x \in F$, the 'powerset' function $y$ is defined by:

$$
\begin{aligned}
\operatorname{dom} y= & \{z: \operatorname{dom} z \rightarrow \bar{\omega} \mid \operatorname{dom} z \subseteq \operatorname{dom} x \wedge \\
& \forall z^{\prime}\left(z^{\prime} \in \operatorname{dom} z \rightarrow z\left(z^{\prime}\right) \leq x\left(z^{\prime}\right)\right\} \text { and } \\
\text { range }(y)= & \{1\} .
\end{aligned}
$$

To show that $y$ is indeed in $F$, let $r(x)=\alpha+1$. To prove that $y \in F$, we first show that dom $y \subseteq F_{\alpha+1}$. If $z \in \operatorname{dom} y$, then dom $z \subseteq d o m x \subseteq F_{\alpha}$ and, therefore, $z \in F_{\alpha+1}$. Hence dom $y \subseteq F_{\alpha+1}$ and, therefore, $y \in F_{\alpha+2}$. Further, since $x \in \operatorname{dom} y, r(y)=\alpha+2$.

$\mathbb{F}$ (Axiom VI) is equivalent to $\forall y($ dom $y \neq \varnothing \rightarrow \exists x(x \in \operatorname{dom} y \wedge \operatorname{dom} x \cap$ dom $y=\varnothing)$ ). Given dom $y \neq \varnothing$, let $x \in \operatorname{dom} y$ be of least rank. If $z \in d o m$ $x$, then $r(z)<r(x)$ and $z \notin$ dom $y$ (since $x$ is of least rank in dom $y$ ). Therefore, dom $x \cap \operatorname{dom} y=\varnothing$ and Axiom VI holds in $\mathbb{F}$.

For Axiom VII, we note that for any $x \in F$ the 'union of $x$ function' $z$ ' is defined as follows:

$\operatorname{dom} z^{\prime}=\cup\{\operatorname{dom} y \mid y \in \operatorname{dom} x\}$ and for each $z \in \operatorname{dom} z^{\prime}$, define $z^{\prime}(z)$ by

(i) $z^{\prime}(z)=\max \{y(z) \mid z \in \operatorname{dom} y \wedge y \in \operatorname{dom} x\}$ if the set $\{y(z) \mid z \in d o m$ $y \wedge y \in \operatorname{dom} x\} \subseteq \bar{\omega}$ is bounded above in $\bar{\omega}$, and

(ii) $z^{\prime}(z)=\min \{y(z) \mid z \in \operatorname{dom} y \wedge y \in \operatorname{dom} x\}$, otherwise.

The function elements in dom $z^{\prime}$ are 'the elements of elements of $x$ ' interpreted in $\mathbb{F}$. To show that $z^{\prime} \in F$, let $r(x)=\alpha+1$. Hence, dom $x \subseteq F_{\alpha}$. Therefore, every $y \in \operatorname{dom} x$ is in $F_{\alpha}$. If $z \in d o m z^{\prime}$ then $r(z)<r(y)$ and $z \in F_{\alpha}$. Since $\operatorname{dom} z^{\prime} \subseteq F_{\alpha}, z^{\prime} \in F_{\alpha+1}$ (and so $z^{\prime} \in F$ ).

Similarly, for Axiom VIII, for any function $x \in F$ the 'additive union of $x$ function' $z^{\prime \prime}$ is defined as follows: dom $z^{\prime \prime}=\operatorname{dom} z^{\prime}=\bigcup\{\operatorname{dom} y \mid y \in \operatorname{dom} x\}$ and for each $z \in \operatorname{dom} z^{\prime \prime}$, define $z^{\prime \prime}(z)$ by

(i) $z^{\prime \prime}(z)=\sum_{y}\{y(z) \cdot x(y) \mid z \in \operatorname{dom} y \wedge y \in \operatorname{dom} x\}$ if the set $\{y \mid z \in$ $\operatorname{dom} y \wedge y \in \operatorname{dom} x\}$ is finite, and

(ii) $z^{\prime \prime}(z)=z^{\prime}(z)$, otherwise. 
The function $z^{\prime \prime}$ is in $F$ by the same argument that established that $z^{\prime}$ is in $F$ (since dom $z^{\prime \prime}=$ dom $z^{\prime}$ ).

To show that the separation schema of MST holds in $\mathbb{F}$, consider a wff $\varphi(x, n)$ of $L$ with free variables including $x$ and $n$ but excluding $n^{\prime}$ and $y$. We have that $\mathbb{F}$ (Axiom $\operatorname{IX}_{\varphi}$ ) is $\forall x \forall n \forall n^{\prime}\left(\left(\varphi^{\prime}(x, n) \wedge \varphi^{\prime}\left(x, n^{\prime}\right)\right) \rightarrow n=n^{\prime}\right) \rightarrow$ $\forall z \exists y \forall x \forall n\left(y(x)=n \leftrightarrow x \in \operatorname{dom} z \wedge n \leq z(x) \wedge \varphi^{\prime}(x, n)\right)$ where $\varphi^{\prime}(x, n)$ is $\mathbb{F}(\varphi(x, n))$, a wff of ZFC, with the same variable symbols as $\varphi(x, n)$. We note that the conjunct $[x]_{n} \subseteq z$ in Axiom $\operatorname{IX}_{\varphi}$ becomes $\{\langle x, n\rangle\} \subseteq z$; that is, $\operatorname{dom}\{\langle x, n\rangle\} \subseteq$ dom $z \wedge\{\langle x, n\rangle\}(x) \leq z(x)$, or simply $x \in \operatorname{dom} z \wedge n \leq z(x)$. Let $\varphi^{\prime}(x, n)$ be such that the antecedent of $\mathbb{F}$ (Axiom IX $\left.\operatorname{IX}_{\varphi}\right)$ holds, and let $z$ be an arbitrary function in $F$. Using separation in ZFC, we define a subset of dom $z$ (which we call dom $y$ ) as follows: dom $y=\{x \in \operatorname{dom} z \mid \exists n(n \in \bar{\omega} \wedge n \leq z(x) \wedge$ $\left.\varphi^{\prime}(x, n)\right\}$. For each $x \in d o m y$, we define $y(x)=n \leftrightarrow \varphi^{\prime}(x, n) \wedge n \leq z(x)$. The function $y$ is well-defined since for every $x \in \operatorname{dom} y$ there is at most one $n$ such that $\varphi^{\prime}(x, n)$ holds (we assumed that $\varphi^{\prime}(x, n)$ is functional). To prove that the function $y$ is in $F$, let $r(z)=\alpha+1$. Then dom $y \subseteq$ dom $z \subseteq F_{\alpha}$ and $y \in F_{\alpha+1}$ as required. Therefore, Axiom $\mathrm{IX}_{\varphi}$ holds in $\mathrm{F}$.

The replacement schema of MST also holds in $\mathrm{F}$. For any replacement axiom $\mathrm{X}_{\varphi}$ of MST, consider its interpretation $\mathbb{F}\left(\right.$ Axiom $\left.X_{\varphi}\right)$ :

$$
\begin{aligned}
& \forall x \forall y \forall y^{\prime}\left(\left(\varphi^{\prime}(x, y) \wedge \varphi^{\prime}\left(x, y^{\prime}\right)\right) \rightarrow y=y^{\prime}\right) \rightarrow \\
& \forall z \exists z^{\prime} \forall y \forall n\left(z^{\prime}(y)=n \leftrightarrow\left[\exists x\left(z(x)=n \wedge \varphi^{\prime}(x, y)\right) \wedge\right.\right. \\
& \left.\left.\forall x \forall m\left(\left(z(x)=m \wedge \varphi^{\prime}(x, y)\right) \rightarrow n \leq m\right)\right]\right)
\end{aligned}
$$

where $\varphi^{\prime}(x, y)$ is $\mathbb{F}(\varphi(x, y))$, a wff of ZFC with free variables including $x$ and $y$ but excluding $y^{\prime}$ and $z^{\prime}$. Let $\varphi^{\prime}(x, y)$ be such that the antecedent of $\mathbb{F}$ (Axiom $\mathrm{X}_{\varphi}$ ) holds, and let $z$ be an arbitrary function in $F$. We apply ZFC replacement to the set dom $z$ using the wff $\varphi^{\prime}(x, y)$ to obtain a new set which we call dom $z^{\prime}: y \in \operatorname{dom} z^{\prime} \leftrightarrow \exists x\left(x \in \operatorname{dom} z \wedge \varphi^{\prime}(x, y)\right)$. We define a function $z^{\prime}$ with domain $\operatorname{dom} z^{\prime}$ as follows: for every $y \in d o m z^{\prime}$, define

$$
\begin{aligned}
z^{\prime}(y)=n \leftrightarrow[ & {\left[\exists x \left(z(x)=n \wedge \varphi^{\prime}(x, y) \wedge\right.\right.} \\
& \left.\forall x \forall m\left(\left(z(x)=m \wedge \varphi^{\prime}(x, y)\right) \rightarrow n \leq m\right)\right] .
\end{aligned}
$$

To show that $z^{\prime} \in F$, let $\beta=\cup\left\{r(y) \mid y \in\right.$ dom $\left.z^{\prime}\right\}$. Every $y \in d o m z^{\prime}$ is in $F_{\beta}$. So $d o m z^{\prime} \subseteq F_{\beta}$ and $z^{\prime} \in F_{\beta+1}$ as required. Therefore Axiom $\mathrm{X}_{\varphi}$ holds in $\mathbb{F}$.

Before we discuss Axiom XI, we note that $x \cup y$ is not, in general, a function in $F$ when both $x$ and $y$ are in $F$. In particular, if there is some $z \in d o m x \cap$ dom $y$ such that $x(z) \neq y(z)$, then $x \cup y$ is not a function (both $\langle z, x(z)\rangle$ and $\langle z, y(z)\rangle$ are elements of $x \cup y)$. This is not the case with $x \cup\{\langle x, 1\rangle\}$ however. If $x \in F$, then $x \cup\{\langle x, 1\rangle\} \in F$ since the domains are disjoint. If dom $x \cap$ $\operatorname{dom}\{\langle x, 1\rangle\}=\operatorname{dom} x \cap\{x\} \neq \varnothing$, then $x \in \operatorname{dom} x$. But $x \in \operatorname{dom} x$ implies that $r(x)<r(x)$, which is absurd. Therefore, the ordered pairs in $x$ together with $\langle x, 1\rangle$ always form a new function in $F$.

The interpretation of Axiom XI in $\mathbb{F}$ is $\exists y(\varnothing \in$ dom $y \wedge \forall x(x \in$ dom $y \rightarrow$ $x \cup\{\langle x, 1\rangle\} \in$ dom $y))$. Put dom $y=\{\hat{n} \mid n \in \omega\}$ which is a set - the image of $\omega$ in $F$ under the embedding '. Since $0 \in \omega, \hat{0}=\varnothing \in$ dom $y$. If $x \in \operatorname{dom} y$ then $x=\hat{n}$ for some $n \in \omega$. If $n \in \omega$, then $n \cup\{n\} \in \omega$ and $n \hat{\cup}\{n\}=\hat{n} \cup\{\langle\hat{n}, 1\rangle\}=$ $x \cup\{\langle x, 1\rangle\} \in$ dom $y$ as required. Since $\mathbb{F}$ (Axiom $\mathrm{XI}$ ) says nothing about the range of $y$, we simply put range $(y)=\{1\}$. By induction one can show that 
$\forall n \hat{n} \in F_{n+1}$. Therefore, every $\hat{n} \in \operatorname{dom} y$ is in $F_{\omega}=\bigcup_{n<\omega} F_{n}$. Since dom $y \subseteq F_{\omega}$, $y \in F_{\omega+1}$, and the function $y$ is in $F$ as required.

For Axiom XII, we consider a function $y$ in $F$ with dom $y \neq \varnothing$ and $\forall x(x \in$ dom $y \rightarrow \operatorname{dom} x \neq \varnothing)$ and $\forall x \forall z((x \in \operatorname{dom} y \wedge z \in \operatorname{dom} y \wedge x \neq z) \rightarrow \operatorname{dom} x \cap$ dom $z \neq \varnothing)$. By replacement in ZFC, the set $\{$ dom $x \mid x \in d o m y\}$ is nonempty, and its elements are nonempty pairwise disjoint sets. By the AC of ZFC there exists a choice set, which we call dom $y^{\prime}$, containing a single function $x^{\prime}$ from each dom $x$. For each such $x^{\prime} \in$ dom $y^{\prime}$ we define $y^{\prime}\left(x^{\prime}\right)=n$ iff $x^{\prime} \in$ dom $x \wedge$ $y(x)=n$. It is easy to show that $y^{\prime}$ is a 'choice function' for the function $y$ as required by $\mathbb{F}$ (Axiom XII). To show that $y^{\prime}$ is in $F$, let $r(y)=\alpha+1$ so that dom $y \subseteq F_{\alpha}$. Since each $x \in d o m y \subseteq F_{\alpha}$, each such $x \in F_{\alpha+1}$ is such that dom $x \subseteq F_{\alpha}$. To show that dom $y^{\prime} \subseteq F_{\alpha}$, if $x^{\prime} \in \operatorname{dom} y^{\prime}$ then $x^{\prime} \in \operatorname{dom} x \subseteq F_{\alpha}$. Therefore, dom $y^{\prime} \subseteq F_{\alpha}$ and $y^{\prime} \in F_{\alpha+1}$ as required.

Theorem For any wff $\varphi$ of $L$, if $M S T \vdash \varphi$ then $Z F C \vdash \mathbb{F}(\varphi)$.

Proof: If $\varphi$ is an axiom of MST, we have shown in every case that $\varphi$ holds in $\mathbb{F}$; that is, $\mathrm{ZFC} \vdash \mathbb{F}(\varphi)$. Since the $\mathbb{F}$-interpretation respects rules of inference, the $\mathbb{F}$-interpretation of a proof of $\varphi$ in MST is a proof of $\mathbb{F}(\varphi)$ in ZFC.

Therefore, the $L$-structure $\mathbb{F}$ is a model of MST. It follows that MST is relatively consistent; that is, if ZFC is consistent, then MST is consistent. If MST $\vdash \psi \wedge \sim \psi$, then $\mathrm{ZFC} \vdash \mathbb{F}(\psi \wedge \sim \psi)$, then $\mathrm{ZFC} \vdash \mathbb{F}(\psi) \wedge \sim \mathbb{F}(\psi)$, a contradiction in $\mathrm{ZFC}$.

In ZFC, we extend the function ${ }^{\wedge}: \omega \rightarrow F$ to the function ${ }^{\wedge}: V \rightarrow F$ as follows: for each $y \in V$, define $\hat{y}=\{\langle\hat{x}, 1\rangle \mid x \in y\}$. For each $y \in V, y \approx \hat{y}$ by the bijection $x \mapsto\langle\hat{x}, 1\rangle$. The range of the extended ' function is the class $\hat{V}=$ $\{\hat{x} \mid x \in V\}$. In fact, $\hat{V}=\bigcup_{\alpha \in O n} \hat{V}_{\alpha}$ where $\hat{V}_{\alpha}=\left\{\hat{x} \mid x \in V_{\alpha}\right\}$ for every $\alpha \in$ On. To prove that $\hat{V} \subset F$, one can prove the

Theorem For every $\alpha \in O n, \hat{V}_{\alpha} \subseteq F_{\alpha}$.

Proof: By induction on the ordinal $\alpha$.

For every wff $\varphi$ of ZFC, we define the reinterpretation of $\varphi$ in $\hat{V}$, denoted by $\varphi^{\hat{V}}$, as follows:

$(x=y)^{\hat{V}}$ is $x \in \hat{V} \wedge y \in \hat{V} \wedge x=y$;

$(x \in y)^{\hat{V}}$ is $x \in \hat{V} \wedge y \in \hat{V} \wedge y(x)=1 ;(\sim \psi)^{\hat{V}}$ is $\sim \psi^{\hat{V}}$;

$(\psi \vee \theta)^{\hat{V}}$ is $\psi^{\hat{V}} \vee \theta^{\hat{V}} ;(\exists x \psi)^{\hat{V}}$ is $\exists x\left(x \in \hat{V} \wedge \psi^{\hat{V}}\right)$; and

$(\forall x \psi)^{\hat{V}}$ is $\forall x\left(x \in \hat{V} \rightarrow \psi^{V}\right)$.

If $\varphi$ is a wff of ZFC, then $\varphi^{\hat{V}}$ is also a wff of ZFC. Diagramatically, for a wff $\varphi$ of ZFC:

$\hat{V}$-reinterpretation

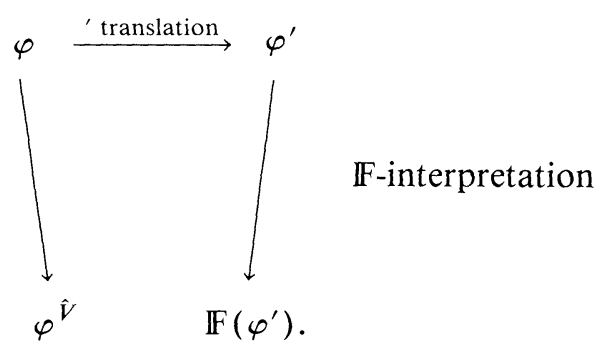


The ' translation was defined on page 57. We wish to prove that ZFC $\vdash \varphi^{\hat{V}} \leftrightarrow$ $\mathbb{F}\left(\varphi^{\prime}\right)$ for every wff $\varphi$ of ZFC. One must first prove the

Theorem For every mset y of MST, ZFC $\vdash \mathbb{F}(H \operatorname{Set}(y)) \leftrightarrow y \in \hat{V}$.

Proof: By induction on the rank of $y$ in $M$ using the equivalence $\operatorname{HSet}(y) \leftrightarrow$ $\operatorname{Set}(y) \wedge \forall x(x \in y \rightarrow H \operatorname{Set}(x))$.

Theorem If $\varphi$ is a wff of ZFC, then $Z F C \vdash \varphi^{\hat{V}} \leftrightarrow \mathbb{F}\left(\varphi^{\prime}\right)$.

Proof: By induction on the logical complexity of $\varphi$. For example, if $\varphi$ is $x \in y$, then $\varphi^{\hat{V}}$ is $(x \in y)^{\hat{V}}$ or $x \in \hat{V} \wedge y \in \hat{V} \wedge y(x)=1 . \mathbb{F}\left((x \in y)^{\prime}\right)$ is $\mathbb{F}(H \operatorname{Set}(x) \wedge$ $\left.H \operatorname{Set}(y) \wedge x \in \in^{1} y\right)$ or $x \in \hat{V} \wedge y \in \hat{V} \wedge y(x)=1$ by the previous theorem. Similarly when $\varphi$ is $x=y$. The induction then proceeds by cases, assuming the equivalence holds for subformulas of $\varphi$.

Corollary For any wff $\varphi$ of $Z F C, Z F C \vdash \varphi^{\hat{V}}$ iff $Z F C \vdash \mathbb{F}\left(\varphi^{\prime}\right)$.

Theorem For any wff $\varphi$ of $Z F C$, if $Z F C \vdash \varphi$ then $Z F C \vdash \varphi \hat{v}$.

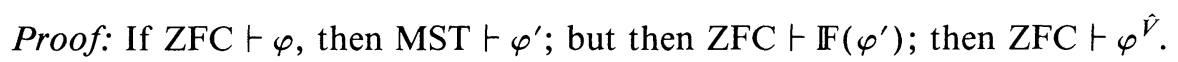

Therefore, the class $\hat{V}$ (a cumulative hierarchy of $\{1\}$-valued functions) is a model of ZFC. The class $\hat{V}$ is also a model of $\mathrm{ZFC}^{\prime}$ if we interpret $\operatorname{HSet}(x) \wedge$ $H \operatorname{Set}(y) \wedge x \in^{1} y$ by $x \in \hat{V} \wedge y \in \hat{V} \wedge y(x)=1$. Therefore, just as the class $F$ of $\bar{\omega}$-valued functions models the mset universe $M$, the subclass $\hat{V}$ of $M$ of $\{1\}$ valued functions models the hset universe $\{x \mid \operatorname{HSet}(x)\}$.

For any wff $\varphi$ of ZFC,

$$
\begin{gathered}
Z F C \vdash \varphi \Rightarrow \operatorname{MST} \vdash \varphi^{\prime} \\
\Downarrow \\
\operatorname{ZFC} \vdash \varphi^{\hat{V}} \Leftrightarrow \operatorname{ZFC} \vdash \mathbb{F}\left(\varphi^{\prime}\right)
\end{gathered}
$$

where the double arrows represent metamathematical implications. To show that all arrows hold in both directions, we prove the following

Theorem For any wff $\varphi$ of $Z F C$,
(i) $Z F C \vdash \varphi \leftrightarrow \varphi^{\hat{V}}$
(ii) if $M S T \vdash \varphi^{\prime}$ then $Z F C \vdash \varphi$ and
(iii) if $Z F C \vdash \mathbb{F}\left(\varphi^{\prime}\right)$ then $M S T \vdash \varphi^{\prime}$.

Proof: (i) By induction on the logical complexity of $\varphi$. Recall that $\hat{y}=\{\langle\hat{x}$, $1\rangle \mid x \in y\}$ so that $\mathrm{ZFC} \vdash x \in y \leftrightarrow\langle\hat{x}, 1\rangle \in \hat{y} \leftrightarrow \hat{y}(\hat{x})=1$. (ii) and (iii) follow from the previous diagram and (i).

Therefore, all arrows hold in both directions in the above diagram.

A theory $T^{\prime}$ is a conservative extension of a theory $T$ if

(i) $L(\mathrm{~T}) \subseteq L\left(\mathrm{~T}^{\prime}\right)$ and

(ii) for every wff $\varphi$ of $L(\mathrm{~T}), \mathrm{T} \vdash \varphi \Leftrightarrow \mathrm{T}^{\prime} \vdash \varphi$.

The $\Rightarrow$ direction is 'extends' and the $\Leftarrow$ direction is 'conservatively'. The theory MST is not a conservative extension of ZFC since the languages are disjoint

$$
L(\mathrm{ZFC})=\{\in\} \text { and } L(\mathrm{MST})=\left[e,{ }^{,}, \mathrm{S},+, \cdot, 0\right\} .
$$


However, the theory MST is a conservative extension of the copy $\mathrm{ZFC}^{\prime}$ of $\mathrm{ZFC}$. The most we can say about MST and ZFC is: for every wff $\varphi$ of $L(Z F C)$,

$$
\mathrm{ZFC} \vdash \varphi \Leftrightarrow \operatorname{MST} \vdash \varphi^{\prime} .
$$

\section{NOTES}

1. A second edition (1986) of Hailperin [12] replaces the word "heap" by the word "multiset". Some readers will be interested in the investigation of combinatorial properties of msubsets of a finite multiset in Ian Anderson's Combinatorics of Finite Sets, Clarendon Press, Oxford, 1987. I am grateful to John Amson for pointing out the use of multisets ( $K$-subsets) in S. Eilenberg, Automata, Languages, and Machines, Vol. A, Academic Press, New York, 1974. T. J. Smiley has pointed out the use of multisets (or "bags") in the theory of Petri nets. See, for example, W. Reisig, Petri Nets: An Introduction, Springer-Verlag, Heidelberg, 1985, and Appendix A in J. L. Peterson, Petri Net Theory and the Modelling of Systems, Prentice-Hall, Englewood Cliffs, N.J., 1981. P. B. Thistlewaite, M. A. McRobbie, and R. K. Meyer make substantial use of the concept of multiset in their Automated Theorem-Proving in NonClassical Logics, Pitman, London, 1988. Possible uses of "bags" in the theory of relational data bases are suggested by R. R. Yager, "On the theory of bags," International Journal of General Systems, Vol. 13 (1986), pp. 23-37. "Bags" are also used in Alan Bundy's The Computer Modelling of Mathematical Reasoning, Academic Press, New York, 1983. Recent work in the theory of multisets includes "A foundation for multiset theory using BCK-linear logic" (M. W. Bunder, Department of Mathematics, University of Wollongong, Australia) and "A geometrical approach to multiset orderings" (U. Martin, Department of Computer Science, Royal Holloway and Bedford New College, University of London) which is to appear in the Journal of Theoretical Computer Science.

\section{REFERENCES}

[1] Barwise, J. (editor), Handbook of Mathematical Logic, North Holland, Amsterdam, 1977.

[2] Barwise, J., Admissible Sets and Structures, Springer-Verlag, Berlin, 1975.

[3] Blizard, W., Generalizations of the Concept of Set: A Formal Theory of Multisets, Doctoral thesis, Mathematical Institute, University of Oxford, 1986.

[4] Cantor, G., Contributions to the Founding of the Theory of Transfinite Numbers, translation, introduction, and notes by P. Jourdain, Dover, New York, 1955.

[5] Chang, C. C. and H. J. Keisler, Model Theory, North Holland, Amsterdam, 1973.

[6] Dedekind, R., Essays on the Theory of Numbers, translated by W. W. Beman, Dover, New York, 1963.

[7] Dershowitz, H. and Z. Manna, "Proving termination with multiset orderings," pp. 188-202 in Automata, Languages and Programming (Sixth Colloquium, Graz, 1979), Lecture Notes in Computer Science 71, Springer, 1979.

[8] Enderton, H., A Mathematical Introduction to Logic, Academic Press, New York, 1972.

[9] Fisher, A., Formal Number Theory and Computability: A Work Book, Oxford Logic Guide 7, Clarendon Press, Oxford, 1982. 
[10] Fraenkel, A., Y. Bar-Hillel, and A. Levy, Foundations of Set Theory, Second revised edition, North Holland, Amsterdam, 1973.

[11] Frege, G., The Foundations of Arithmetic, translated by J. L. Austin, Second revised edition, Basil Blackwell, Oxford, 1980.

[12] Hailperin, T., Boole's Logic and Probability, North Holland, Amsterdam, 1976.

[13] Hallett, M., Cantorian Set Theory and Limitation of Size, Oxford Logic Guide 10, Clarendon Press, Oxford, 1984.

[14] Halmos, P., Naive Set Theory, Springer-Verlag, Berlin, 1974.

[15] Hickman, J. L., "A note on the concept of multiset," Bulletin of the Australian Mathematical Society, vol. 22 (1980), pp. 211-217.

[16] Kamke, E., Theory of Sets, translated from the Second German Edition by F. Bagemihl, Dover, New York, 1950.

[17] Knuth, D., The Art of Computer Programming, Volume 2, Seminumerical Algorithms, Second edition, Addison-Wesley, Reading, Massachusetts, 1981.

[18] Knuth, D., The Art of Computer Programming, Volume 3, Sorting and Searching, Addison-Wesley, Reading, Massachusetts, 1973.

[19] Lake, J., "Sets, fuzzy sets, multisets and functions," Journal of the London Mathematical Society (2), vol. 12 (1976), pp. 323-326.

[20] Leibniz, G. W., Philosophical Writings, translated by M. Morris and G. H. R. Parkinson, edited by G. H. R. Parkinson, Everyman's Library, J. M. Dent and Sons, London, 1973.

[21] Levy, A., Basic Set Theory, Springer-Verlag, Berlin, 1979.

[22] Manna, Z. and R. Waldinger, The Logical Basis of Computer Programming, Volume 1, Addison-Wesley, Reading, Massachusetts, 1985.

[23] Meyer, R. K. and M. A. McRobbie, "Multisets and relevant implication I and II," Australasian Journal of Philosophy, vol. 60 (1982), pp. 107-139, 265-281.

[24] Monro, G. P., "The concept of multiset," Zeitschrift für mathematische Logik und Grunlagen der Mathematik, to appear.

[25] Parker-Rhodes, A. F., The Theory of Indistinguishables, D. Reidel, Dordrecht, 1981.

[26] Rado, R., "The cardinal module and some theorems on families of sets," Annali di Mathematica Pura ed Applicata, (4), vol. 102 (1975), pp. 135-154.

[27] Weyl, H., Philosophy of Mathematics and Natural Science, Revised and Augmented English Edition, Atheneum, New York, 1963.

[28] Whitney, H., "Characteristic functions and the algebra of logic," Annals of Mathematics, vol. 34 (1933), pp. 405-414.

Department of Pure Mathematics and Mathematical Statistics

Cambridge University

16 Mill Lane

Cambridge CB2 $1 S B$

United Kingdom 